\title{
ANALYSE DES EXPÉRIENCES DE GÉNÉRATION DE SECOND HARMONIQUE
}

\author{
D. CHEMLA et P. KUPECEK \\ Centre National d'Etudes des Télécommunications, 92, Bagneux
}

(Reçu le 19 octobre 1970)

\begin{abstract}
Résumé. - Dans cet article, nous développons une étude détaillée des expériences de génération de second harmonique, étude où sont pris en considération le diamètre fini du faisceau laser focalisé et la géométrie de l'échantillon cristallin, en considérant le matériau dans la situation habituelle où le milieu environnant est l'air. Ceci amène à tenir compte des conditions aux limites sur les deux faces de l'échantillon supposé prismatique et formé d'un matériau qui peut éventuellement être biréfringent et absorbant.
\end{abstract}

\begin{abstract}
In this paper, we carry out a detailed study of second harmonic generation experiments, taking into account the limited beam diameter of the focused laser beam and the crystal sample shape. We consider the material in the usual case where the surrounding medium is air. Then, one is led to write the boundaries conditions on the two limiting surfaces of the sample supposed wedge shaped. The non-linear medium may be birefringent and absorbing.
\end{abstract}

Introduction. - Si les susceptibilités du deuxième ordre interviennent dans un grand nombre de phénomènes, puisqu'elles décrivent le premier des effets non linéaires, seules les études de génération de second harmonique donnent en pratique un moyen de les déterminer. Le principe des expériences de génération de second harmonique est très simple (Fig. 1). Un échantillon de matériau non linéaire soumis à un rayonnement de pulsation $\omega$, émet une lumière de pulsation $2 \omega$, il suffit de séparer l'harmonique du fondamental pour étudier le processus.

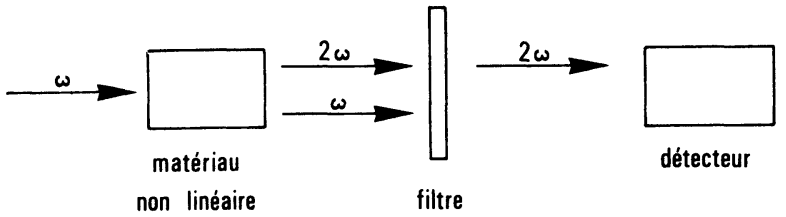

FIG. 1. - Principe des expériences de génération de second harmonique.

Les premiers, Bloembergen et Pershan [1] ont analysé dans le détail les effets produits par une onde plane d'extension infinie tombant sur un demi-espace de matériau non linéaire séparé du vide par un dioptre plan. Il ressort de leurs travaux que le module du vecteur de Poynting de l'onde harmonique créée dans le matériau à une distance $z$ du dioptre est :

$$
\left|S_{2 \omega}\right|=K \mathrm{~d}_{\text {eff }}^{2}\left|E_{\omega}\right|^{2} \frac{\sin ^{2}\left(\frac{\pi z}{2 l_{c}}\right)}{\left(\frac{\pi}{2 l_{c}}\right)^{2}} .
$$

Dans cette formule, $d_{\text {eff }}$ est la valeur efficace de la susceptibilité non linéaire (liée au tenseur $\underline{\underline{\underline{\chi}}}(2)$ et à l'orientation relative des axes cristallins et du vecteur polarisation de l'onde fondamentale $\bar{E}_{\omega}$ ).

$$
l_{c}=\lambda / 4\left[n_{2 \omega}-n_{\omega}\right]
$$

est la longueur de cohérence.

La situation idéale étudiée par Bloembergen et Pershan leur permit d'énoncer les principales lois régissant le processus de génération de second harmonique. Pour décrire la structure fine des résultats d'une expérience réelle, il faut particulariser les hypothèses afin de tenir compte de la nature effective des systèmes en interaction.

Ainsi, Boyd, Kleinman et al. [2, 3, 4] et Bjorkholm [5] ont dû, pour expliquer certaines de leurs expériences, tenir compte de la structure du faisceau fondamental et ont développé un formalisme des interactions non linéaires des faisceaux gaussiens focalisés (représentant bien un faisceau laser dans le mode $\mathrm{TEM}_{00}$ ). Ils ont cependant supposé leurs échantillons immergés dans un liquide de même indice pour ne pas avoir à tenir compte des conditions aux limites.

Dernièrement, Jerphagnon et Kurtz [6], à l'occasion d'expériences très précises sur des matériaux importants qui servent généralement d'étalons pour les mesures comparatives, ont étudié les effets non linéaires dans une lame à faces parallèles d'extension infinie, plongée dans le vide et soumise à un rayonnement d'onde plane en tenant compte des conditions aux limites, sur les deux faces de l'échantillon. Cette analyse s'est révélée absolument nécessaire à l'exploitation correcte de leurs expériences.

Nous avons réalisé une variante de l'expérience de 
Maker [7] proposée par Jerphagnon [8] et utilisé des échantillons prismatiques d'angle au sommet assez important. Nos résultats expérimentaux, en désaccord avec les analyses rappelées ci-dessus nous ont conduits à reconsidérer certains points, en particulier les effets dus à l'extension finie des faisceaux.

Dans le présent article, nous exposerons les études que nous avons été amenés à développer pour expliquer nos expériences. Nous considérerons des configurations de complexité croissante. Ainsi, la première partie se rapportera à une situation particulièrement simple qui nous permettra de décrire les phénomènes fondamentaux. Dans ce cas, que nous nous sommes efforcés de réaliser en pratique, l'échantillon prismatique de matériau non linéaire a la symétrie $\overline{4} 3 \mathrm{~m}$ et l'arête du prisme est un axe 111 parallèle à la polarisation du laser, le diélectrique étant supposé transparent aux deux fréquences fondamentale et harmonique ; puis, nous étendrons notre analyse à des matériaux absorbants et biréfringents.

Nous supposons que le faisceau laser est constitué, du moins dans la région d'interaction, par un pinceau cylindrique d'ondes planes parallèles (voir Annexe $\mathrm{n}^{\circ} 1$ pour la justification de cette hypothèse).

Notations. - Vecteur : $\overline{\mathrm{A}}$; Tenseur du deuxième ordre: $\underline{\underline{A}}$; Tenseur du troisième ordre : A.

Vecteur unitaire $: \hat{\mathrm{v}}$ (vecteur unitaire $\overline{\overline{\text { suivant les }}}$ axes : $\hat{x} \hat{y} \hat{z}$ ).

Variable X dépendant de la pulsation $\Omega: \mathrm{X}_{\Omega}$.

Phase d'une onde plane :

$$
\Phi=\bar{k} \bar{r}-\Omega t=n_{\Omega} \frac{\Omega}{c} \widehat{s} \bar{r}-\Omega t .
$$

Angles d'incidence, de réflexion et de réfraction sur le premier dioptre $: \theta, \theta^{R}, \theta^{\prime}$.

Angles d'incidence, de réflexion et de réfraction sur le deuxième dioptre : $\varphi^{\prime}, \varphi^{\prime R}, \varphi^{\prime \prime}$.

Coefficients de transmission pour l'onde fondamentale : $t_{\omega}, t_{\|}, t_{\perp}$. Coefficients de transmission pour les ondes harmoniques : $T_{\omega}, T_{2 \omega}$. Susceptibilité complexe d'un matériau absorbant :

$$
\chi^{0}=\chi_{\Omega}^{\prime}+i \chi_{\Omega}^{\prime \prime} .
$$

Indice complexe d'un matériau absorbant :

$$
n_{\Omega}^{0}=n_{\Omega}\left(1+i \kappa_{\Omega}\right) \text {. }
$$

Indices ordinaire et extraordinaire d'un matériau biréfringent $: n_{\Omega}^{\mathrm{o}}, n_{\Omega}^{\mathrm{e}}$.

Angle entre la direction de propagation de la phase et celle de l'énergie pour les ondes forcées ou pour les ondes libres extraordinaires $: \rho$. Coefficient d'absorption en intensité lumineuse :

$$
a_{\Omega}=\frac{2 \Omega}{c} n_{\Omega} \kappa_{\Omega} .
$$

Coefficient d'absorption en intensité suivant la normale au dioptre : $\gamma_{\Omega}=(2 \Omega / c)\left(n_{\Omega} \kappa_{\Omega} / \cos \theta_{\Omega}^{\prime}\right)$.

Rencontrant souvent la séquence « vide-diélectrique- vide ", nous faisons, pour distinguer la région où l'on considère la variable $\mathrm{X}$, la convention suivante :

$$
\begin{array}{ccc}
\text { vide } & \text { diélectrique } & \text { vide } \\
\mathrm{X} & \mathrm{X}^{\prime} & \mathrm{X}^{\prime \prime}
\end{array}
$$

\section{PREMIÈRE PARTIE}

I. Expression du champ harmonique dans le cadre de l'approximation paramétrique. - Dans un matériau non linéaire soumis aux champs $E_{\omega}^{\prime}(\bar{r}, t)$ et $\bar{E}_{2 \omega}^{\prime}(\bar{r}, t)$ de pulsation respective $\omega$ et $2 \omega$, apparaissent des ondes forcées de polarisation dont les expressions sont :

$$
\begin{aligned}
& \bar{P}_{2 \omega}^{\mathrm{NLS}}=\hat{p} P_{2 \omega}=\chi^{(2)}(2 \omega=\omega+\omega): \bar{E}_{\omega}^{\prime}(\bar{r}, t) \otimes \bar{E}_{\omega}^{\prime}(\bar{r}, t) \\
& \bar{P}_{\omega}^{\mathrm{NLS}}=\hat{q} P_{\omega}=\chi^{(2)}(\omega=2 \omega-\omega): \bar{E}_{2 \omega}^{\prime}(\bar{r}, t) \otimes{\overline{E_{\omega}}}_{\omega}^{\prime *}(\bar{r}, t) .
\end{aligned}
$$

Ces ondes de polarisation couplent dans le milieu les deux champs qui sont alors solutions d'un système d'équations de Maxwell couplées :

$$
\nabla \times \overline{\mathrm{V}} \times \bar{E}_{\Omega}^{\prime}(r, t)+\frac{\varepsilon_{\Omega}}{c^{2}} \frac{\partial^{2}}{\partial t^{2}} \bar{E}_{\Omega}^{\prime}(r, t)=-\frac{4 \pi}{c^{2}} \frac{\partial^{2}}{\partial t^{2}} \bar{P}_{\Omega}^{\mathrm{NLS}}
$$

avec

$$
\Omega=\omega \quad \text { ou } 2 \omega .
$$

L'un des problèmes fondamentaux de l'optique non linéaire est de déterminer les solutions de ce système compte tenu des différentes contraintes possibles (essentiellement les conditions aux limites).

Dans un cas particulier, très important en pratique, il est possible de trouver une méthode simple pour résoudre ce système. A cause de l'extrême petitesse des susceptibilités non linéaires, on peut négliger le terme de polarisation non linéaire de l'équation de l'onde fondamentale et considérer qu'elle se propage librement dans le matériau. Alors le terme de polarisation non linéaire de l'équation de l'onde harmonique est parfaitement déterminé. La solution la plus générale de l'équation (2) pour la pulsation $2 \omega$ est la somme de la solution générale de l'équation sans deuxième membre et d'une solution particulière de l'équation avec deuxième membre.

Lorsque l'onde excitatrice à la pulsation $\omega$ est une onde plane :

$$
\bar{E}_{\omega}^{\prime}(\bar{r}, t)=\hat{e}^{\prime} E^{\prime} \exp \left(i \Phi_{\omega}^{\prime}\right)
$$

où nous avons posé :

$\hat{e}^{\prime}=$ vecteur unitaire de polarisation,

$E^{\prime}=$ module du champ,

$$
\Phi_{\omega}^{\prime}=\bar{k}^{\prime} \bar{r}-\omega t=n_{\omega} \frac{\omega}{c} \hat{s^{\prime}} \bar{r}-\omega t,
$$

$\hat{s}^{\prime}=$ vecteur unitaire dans la direction du vecteur d'onde.

La relation (1) montre que la polarisation à la pulsation $2 \omega$ est une onde plane de phase :

$$
2 \Phi_{\omega}^{\prime}=2 \bar{k}^{\prime} \bar{r}-2 \omega t \text {. }
$$


La solution générale de l'équation de Maxwell de l'onde harmonique peut se mettre sous la forme :

$$
\begin{aligned}
\bar{E}_{2 \omega}^{\prime}(r, t)=\left(\sum_{l} \hat{e}_{l}^{\prime} E_{l}^{\prime} \exp \left(i \bar{k}_{l}^{\prime} \bar{r}\right)\right. & \left.+\hat{e}_{f}^{\prime} E_{f}^{\prime} \exp \left(i \bar{k}_{f}^{\prime} \bar{r}\right)\right) \\
& \times \exp (-2 i \omega t)
\end{aligned}
$$

où les indices $l$ et $f$ permettent de distinguer les ondes libres solutions de l'équation sans deuxième membre, de l'onde forcée solution de l'équation inhomogène. Bloembergen et Pershan [1] ont montré que, pour les matériaux isotropes :

$$
\begin{gathered}
E_{f}^{\prime} \hat{e}_{f}^{\prime}=\left(\hat{p}-4 \frac{\bar{k}_{\omega}^{\prime} \hat{p}}{\left|\bar{k}_{2 \omega}^{\prime}\right|^{2}} \bar{k}_{\omega}^{\prime}\right) \frac{4 \pi P_{2 \omega}}{n_{\omega}^{2}-n_{2 \omega}^{2}} \\
\bar{k}_{f}^{\prime}=2 \bar{k}_{\omega}^{\prime}=\frac{2 \omega}{c} n_{\omega} \widehat{s}_{f}^{\prime} \\
\bar{k}_{l}^{\prime}=\bar{k}_{2 \omega}^{\prime}=\frac{2 \omega}{c} n_{2 \omega} \widehat{s}_{l}^{\prime}
\end{gathered}
$$

(Pour démonstration de la formule (4a), voir l'Annexe $n^{\circ} 2$ ). L'amplitude, la polarisation et la direction des ondes libres sont déterminées par les conditions aux limites à la surface du diélectrique.

Nous étudierons dans le détail une configuration que nous nous sommes efforcés de réaliser dans nos expériences. Cet exemple nous permettra d'aborder de manière simple les principaux phénomènes. Nous étendrons ensuite nos résultats à des situations plus complexes.

Nous considérons des échantillons de matériaux transparents aux fréquences $\omega$ et $2 \omega$ ne présentant pas d'activité optique et tels que la polarisation non linéaire soit parallèle au champ électrique fondamental. On peut par exemple réaliser cette dernière condition en choisissant des matériaux de symétrie ponctuelle $\overline{4} 3 \mathrm{~m}$, les échantillons étant taillés de manière à ce qu'un axe 111 soit dans le plan du premier dioptre et orienté parallèlement au vecteur champ électrique de l'onde fondamentale (Fig. 2).

Soit $d_{\text {eff }}$ le coefficient non linéaire efficace, alors :

$$
\begin{gathered}
\left.\bar{k}_{\omega}^{\prime} \hat{p}=0 \hat{(s} \perp \hat{p}\right) \quad P_{2 \omega}=\mathrm{d}_{\text {eff }}\left|\bar{E}_{\omega}^{\prime}\right|^{2} \\
\hat{e}_{f}^{\prime}=\hat{p} \quad E_{f}^{\prime}=4 \pi \frac{\mathrm{d}_{\text {eff }}}{n_{\omega}^{2}-n_{2 \omega}^{2}}\left|\bar{E}_{\omega}^{\prime}\right|^{2} .
\end{gathered}
$$

Lors de la traversée de l'échantillon, l'onde fondamentale rencontre deux dioptres plans : le premier, à l'entrée dans le cristal, du type "vide-matériau non linéaire ", le second, du type "matériau non linéairevide $»$.

Les conditions aux limites imposées aux ondes lors de la traversée des différents dioptres sont de deux types :

a) Conditions aux limites relatives aux vecteurs d'onde : pour qu'une condition écrite en un point d'un dioptre sur une variable dont la phase est $\Phi=\bar{k} \bar{r}-\omega t$

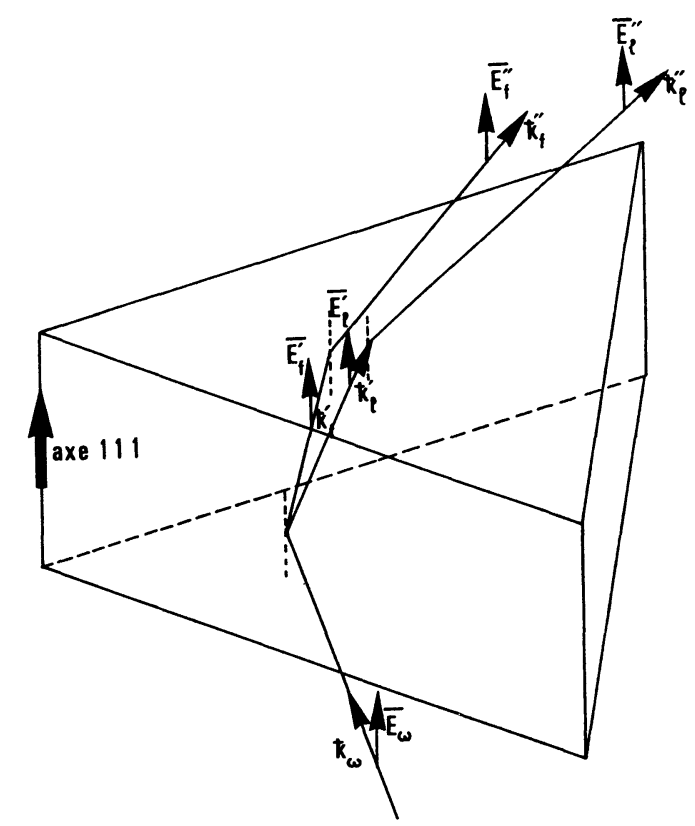

FIG. 2. - Polarisation des champs fondamentaux et harmoniques dans l'échantillon cristallin.

soit valable en tout point d'un dioptre, il faut que toutes les ondes arrivant sur le dioptre aient même composante tangentielle du vecteur d'onde :

$$
\bar{k}_{T}=\mathrm{C}^{\mathrm{te}} \text {. }
$$

Cette condition permet de déterminer les directions possibles de propagation pour les ondes réfléchie et réfractée à la surface du dioptre. En optique linéaire, elle implique les lois de Descartes. En optique non linéaire, elle détermine les vecteurs de propagation des ondes planes apparaissant dans l'équation (3).

b) Conditions aux limites relatives aux composantes tangentielles des champs :

$$
\bar{E}_{T}=\mathrm{C}^{\mathrm{te}} \quad \bar{H}_{T}=\mathrm{C}^{\mathrm{te}} .
$$

Ces conditions impliquent l'existence des ondes réfléchie et réfractée dont elles déterminent les amplitudes ; en optique linéaire elles induisent les lois de Fresnel.

II. Conditions aux limites d'un dioptre «vidematériau non linéaire ». - Le fait qu'à chaque instant $\bar{E}_{T}$ et $\bar{H}_{T}$ soient continus à la traversée de ce dioptre implique que les conditions aux limites doivent être satisfaites séparément pour les deux fréquences (voir Fig. 3).

1) ONDE FONDAMENTALE $-a$ ) La condition sur les vecteurs d'onde conduit aux lois de Descartes :

$$
\theta_{\omega}^{R}=-\theta_{\omega} \quad \sin \theta_{\omega}=n_{\omega} \sin \theta_{\omega}^{\prime} .
$$

b) La condition sur les composantes tangentielles des champs pour un vecteur champ électrique perpendiculaire au plan d'incidence conformément à 


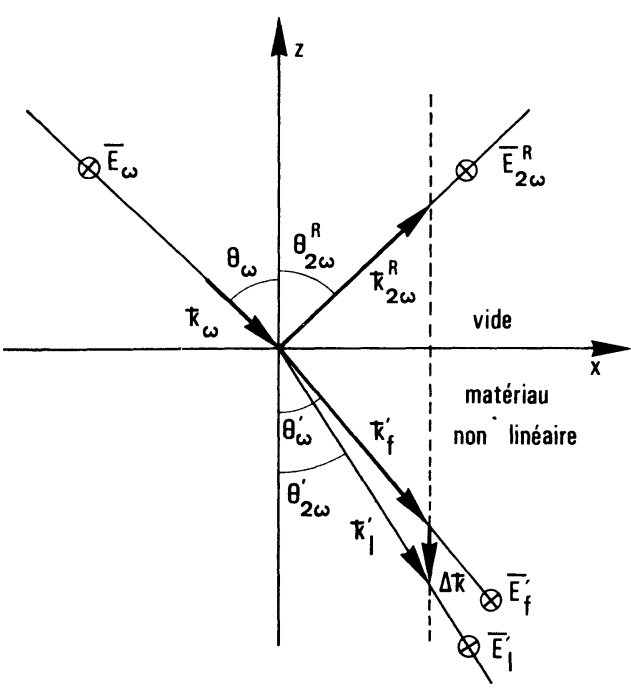

Fig. 3. - Définition des symboles sur le dioptre vide-matériau non linéaire pour les ondes fondamentales et harmoniques.

l'orientation choisie (Fig. 2 et 3 ) conduit aux lois de Fresnel :

$$
\begin{array}{ll}
E_{\omega}^{\prime}=t_{\omega} E_{\omega} & E_{\omega}^{R}=r_{\omega} E_{\omega} \\
t_{\omega}^{\top}=\frac{2 \cos \theta_{\omega}}{\cos \theta_{\omega}+n_{\omega} \cos \theta_{\omega}^{\prime}} & r_{\omega}=\frac{\cos \theta_{\omega}-n_{\omega} \cos \theta_{\omega}^{\prime}}{\cos \theta_{\omega}+n_{\omega} \cos \theta_{\omega}^{\prime}} .
\end{array}
$$

2) ONDE HARMONIQUE. - Nous avons vu que dans l'approximation des petits transferts d'énergie le rayonnement à la fréquence $2 \omega$ dans le milieu non linéaire est représenté par la somme d'une onde forcée et d'ondes libres. L'onde forcée est parfaitement définie en polarisation, module et phase par la connaissance de l'onde fondamentale et des propriétés linéaires et non linéaires du matériau. D'après la condition aux limites relative aux vecteurs d'onde, seules les ondes planes ayant même $k_{T}$ que l'onde fondamentale incidente peuvent se propager soit dans le vide soit dans le milieu non linéaire.

Pour respecter le principe de causalité, on ne retient qu'une onde se propageant dans le sens des $z$ croissants dans le vide et une onde se propageant dans le sens des $z$ décroissants dans le matériau (Fig. 3).

Les conditions aux limites sur les composantes tangentielles des champs définissent la polarisation et l'amplitude de ces deux ondes. Chacune d'elles est définie par les deux composantes de son champ électrique. Les conditions aux limites s'expriment par quatre équations linéaires scalaires de ces composantes de champ électrique. Le problème des conditions aux limites est ainsi résolu par l'existence d'une onde libre dans le matériau et aussi d'une onde harmonique "réfléchie ", onde qui est créée dans une épaisseur de l'ordre de quelques longueurs d'onde qui constitue ce qu'on appelle physiquement «la surface du diélectrique $"$.

Dans la disposition que nous avons choisie (voir Fig. 3), toutes les ondes se propageant dans le plan d'incidence défini par l'onde fondamentale à l'extérieur, et elles ont même polarisation, perpendiculaire au plan d'incidence. Le champ à la pulsation $2 \omega$ est alors donné par :

$$
\bar{E}_{2 \omega}^{\prime}=\widehat{e}_{l}^{\prime} E_{l}^{\prime} \exp \left(\overline{i k_{l}^{\prime}} \bar{r}\right)+\widehat{e}_{f}^{\prime} E_{f}^{\prime} \exp \left(i \bar{k}_{f}^{\prime} \bar{r}\right)
$$

à l'intérieur du matériau, et par :

$$
\bar{E}_{2 \omega}^{R}=\hat{e}_{R} E_{R} \exp \left(i \bar{k}_{R} \bar{r}\right)
$$

dans le vide, à l'extérieur.

Les directions de ces ondes sont définies par :

$$
\begin{array}{ll}
\sin \theta_{\omega}^{\prime}=\frac{1}{n_{\omega}} \sin \theta_{\omega} & \sin \theta_{2 \omega}^{\prime}=\frac{1}{n_{2 \omega}} \sin \theta_{\omega} \quad \theta^{R}=-\theta_{\omega} \\
\left|\bar{k}_{f}^{\prime}\right|=n_{\omega} \frac{2 \omega}{c} & \left|\bar{k}_{l}^{\prime}\right|=n_{2 \omega} \frac{2 \omega}{c} \quad\left|k_{2 \omega}^{R}\right|=\frac{2 \omega}{c} .
\end{array}
$$

Notons dès à présent un point fondamental sur lequel nous reviendrons très souvent: la différence des vecteurs d'onde des ondes créées dans le diélectrique est portée par la normale au dioptre vidematériau non linéaire.

$$
\overline{\Delta k}=\bar{k}_{l}^{\prime}-\bar{k}_{f}^{\prime} \text { est parallèle à l'axe oz . }
$$

Finalement, l'expression des diverses amplitudes est :

$$
\begin{aligned}
& \int E_{l}^{\prime}=A_{l} E_{f}^{\prime}=-\frac{\cos \theta_{\omega}+n_{\omega} \cos \theta_{\omega}^{\prime}}{\cos \theta_{\omega}+n_{2 \omega} \cos \theta_{2 \omega}^{\prime}} E_{f}^{\prime} \\
& E_{2 \omega}^{R}=A_{R} E_{f}^{\prime}=\frac{n_{2 \omega} \cos \theta_{2 \omega}^{\prime}-n_{\omega} \cos \theta_{\omega}^{\prime}}{n_{2 \omega} \cos \theta_{2 \omega}^{\prime}+\cos \theta_{\omega}} \\
& \bar{E}_{2 \omega}^{\prime}=\widehat{y} 4 \pi \frac{\mathrm{d}_{\text {eff }} t_{\omega}^{2}\left|\bar{E}_{\omega}\right|^{2}}{n_{\omega}^{2}-n_{2 \omega}^{2}} \\
& \times\left(\exp \left(i \bar{k}_{f}^{\prime} \bar{r}\right)-\frac{\cos \theta_{\omega}+n_{\omega} \cos \theta_{\omega}^{\prime}}{\cos \theta_{\omega}+n_{2 \omega} \cos \theta_{2 \omega}^{\prime}} \exp \left(\overline{i k}_{l} \bar{r}\right)\right) .
\end{aligned}
$$

REMARQUE. - Nous pouvons dès à présent étendre nos résultats au cas de l'accord de phase, c'est-à-dire lorsque l'on réalise la condition $n_{\omega}=n_{2 \omega}$. Nous pouvons exprimer le champ harmonique réfléchi et le champ harmonique dans le matériau sous la forme :

$$
\begin{aligned}
\bar{E}_{2 \omega}^{\prime}=\bar{E}_{l}^{\prime}+\bar{E}_{f}^{\prime}=\hat{y} 4 \pi \mathrm{d}_{\mathrm{eff}} \frac{t_{\omega}^{2}\left|E_{\omega}\right|^{2}}{n_{\omega}+n_{2 \omega}} \\
\times \frac{1+A_{l} \exp \left(i \frac{2 \omega}{c}\left(n_{2 \omega} \hat{s}_{l}^{\prime}-n_{\omega} \hat{s}_{f}^{\prime}\right) \bar{r}\right)}{n_{\omega}-n_{2 \omega}} \\
\times \exp \left(i \frac{2 \omega}{c} n_{\omega} \hat{s}_{f}^{\prime} \bar{r}\right) \\
\bar{E}_{2 \omega}^{R}=\hat{y} 4 \pi \mathrm{d}_{\mathrm{eff}} \frac{t_{\omega}^{2}\left|E_{\omega}\right|^{2}}{n_{\omega}+n_{2 \omega}} \frac{A_{R} \exp \left(i \frac{2 \omega}{c} \hat{s}_{R} \bar{r}\right)}{n_{\omega}-n_{2 \omega}}
\end{aligned}
$$

Posons :

$$
n_{2 \omega}-n_{\omega}=\delta n \quad \bar{r}=X \hat{x}+\hat{Y y}+\hat{Z z} .
$$


En développant au premier ordre en $\delta n$

on trouve :

$$
A_{l}, A_{R} \text { et } \exp \left(i \frac{2 \omega}{c}\left(n_{2 \omega} \hat{s}_{l}^{\prime}-n_{\omega} \hat{s}_{f}^{\prime}\right) \bar{r}\right)
$$

$$
\begin{aligned}
A_{l} & \simeq-1+\frac{\delta n}{\cos \theta_{\omega}^{\prime}\left(\cos \theta_{\omega}+n_{\omega} \cos \theta_{\omega}^{\prime}\right)} \\
A_{R} & \simeq \frac{\delta n}{\cos \theta_{\omega}^{\prime}\left(\cos \theta_{\omega}^{\prime}+n_{\omega} \cos \theta_{\omega}^{\prime}\right)} \\
& \quad \exp \left(i \frac{2 \omega}{c}\left(n_{2 \omega} \hat{s}_{l}^{\prime}-n_{\omega} \hat{s}_{f}^{\prime}\right) \bar{r}\right) \simeq 1+i \frac{4 \pi}{\lambda_{\omega}} z \frac{\delta n}{\cos \theta_{\omega}^{\prime}} .
\end{aligned}
$$

L'accord de phase est obtenu lorsque $\delta n$ tend vers zéro. Les champs harmoniques ont des limites finies.

$$
\begin{gathered}
\bar{E}_{2 \omega}^{\prime}=-\hat{y} 4 \pi \mathrm{d}_{\text {eff }} \frac{t_{\omega}^{2}\left|E_{\omega}\right|^{2}}{2 n_{\omega} \cos \theta_{\omega}^{\prime}} \\
\times\left(\frac{1}{\cos \theta+n_{\omega} \cos \theta_{\omega}^{\prime}}-i \frac{4 \pi}{\lambda_{\omega}} z\right) \exp \left(i \frac{2 \omega}{c} n_{\omega} \widehat{s}_{f}^{\prime} \bar{r}\right) \\
\bar{E}_{\omega}^{R}=-\hat{y} 4 \pi \mathrm{d}_{\text {eff }} \frac{t_{\omega}^{2}\left|E_{\omega}\right|^{2}}{2 n_{\omega} \cos \theta_{\omega}^{\prime}} \frac{1}{\cos \theta+n_{\omega} \cos \theta_{\omega}^{\prime}} \\
\times \exp \left(i \frac{2 \omega}{c} \widehat{s}_{R} \bar{r}\right) .
\end{gathered}
$$

Dans le matériau, le champ harmonique est la somme de deux termes. Le premier, égal en amplitude au champ réfléchi, traduit les effets de surface. Le second, linéairement croissant, est prépondérant dès que la profondeur dépasse quelques longueurs d'onde. Il correspond à l'onde amplifiée pour laquelle la théorie exacte [9] prévoit une variation en th $z$, soit pour les petits transferts d'énergie (l'approximation qui est la nôtre), une croissance linéaire avec la profondeur.

III. Conditions aux limites d'un dioptre matériau non linéaire-vide. - Les deux composantes de l'onde harmonique sont de nature fondamentalement différente. Si la solution de l'équation homogène est une onde libre qui une fois créée à la surface du premier dioptre se propage librement en suivant les lois de l'optique classique, l'onde forcée n'a d'existence que dans la mesure où l'onde excitatrice est présente. Il faut donc écrire les conditions aux limites sur le deuxième dioptre indépendamment pour chacune des composantes. Ce point n'est pas gênant : on peut en effet envisager des configurations particulières où l'une des ondes peut être éliminée par exemple par absorption préférentielle (voir deuxième partie). Toujours dans le même ordre d'idées, Bloembergen et al. ont montré [9] que les comportements de ces deux ondes en ce qui concerne leurs angles de réfraction limite étaient totalement différents.

Dans les paragraphes qui suivent, nous désignerons par $\varphi^{\prime}$ les angles d'incidence sur le deuxième dioptre ; ils sont liés aux angles $\theta^{\prime}$ correspondants et à l'angle dièdre des deux dioptres par $S=\varphi^{\prime}+\theta^{\prime}$. Les phases de toutes les ondes qui apparaissent sont déterminées par celle de l'onde fondamentale incidente. Comme origine $\mathrm{O}$ on a pris un point du premier dioptre ; pour tenir compte dans les calculs ultérieurs des différences de phase entre les différentes onces, l'écriture des conditions aux limites en un point $\mathrm{Mdu}$ deuxième dioptre doit nécessairement faire apparaître le terme de phase de chaque onde.

1) Conditions aUX limites pour L'ONDE LiBRe. Cette onde suit les lois classiques de l'optique : à la traversée du dioptre elle crée une onde réfléchie dans le diélectrique et une onde réfractée dans le vide (Fig. 4) : $\bar{E}_{l}^{\prime}$ donne naissance à $\bar{E}_{l}^{\prime R}$ et à $\bar{E}_{l}^{\prime \prime}$. Les lois de Descartes s'écrivent :

${\overline{E_{l}}}_{l}^{\prime R}\left\{\begin{array}{l}\varphi_{2 \omega}^{\prime R}=-\varphi_{2 \omega}^{\prime} \\ \left|\bar{k}_{l}^{\prime R}\right|=n_{2 \omega} \frac{2 \omega}{c}\end{array} \quad \bar{E}_{l}^{\prime \prime}\left\{\begin{array}{l}\sin \varphi_{2 \omega}^{\prime \prime}=n_{2 \omega} \sin \varphi_{2 \omega}^{\prime} \\ \left|\bar{k}_{l}^{\prime \prime}\right|=\frac{2 \omega}{c} .\end{array}\right.\right.$

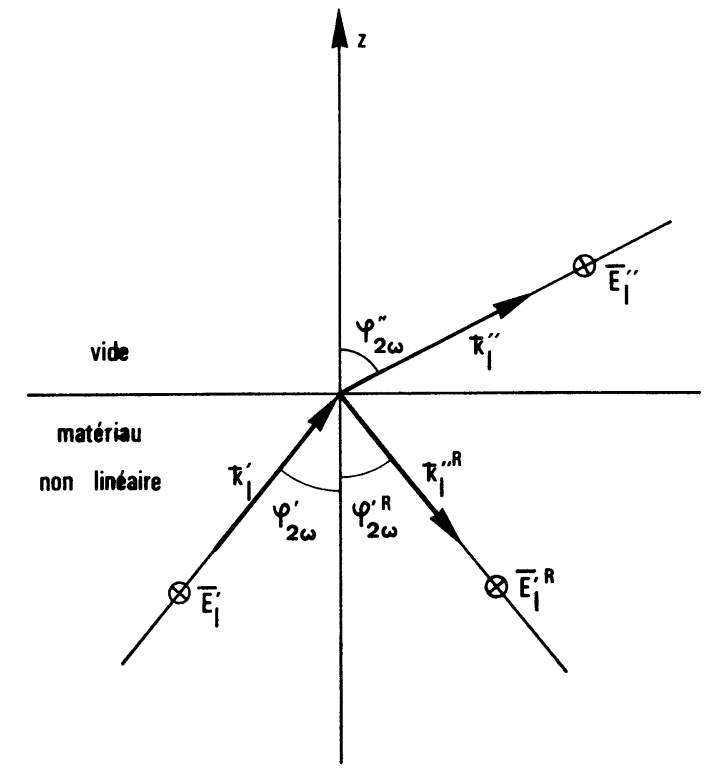

Fig. 4. - Définition des symboles sur le dioptre matériau non linéaire-vide pour l'onde harmonique libre.

Les lois de Fresnel écrites au point $\mathrm{M}$ du deuxième dioptre $\overline{\mathrm{OM}}=\overline{\mathrm{R}}$ donnent :

$$
\begin{gathered}
E_{l}^{\prime \prime} \exp \left(i \bar{k}_{l}^{\prime \prime} \bar{R}\right)=T_{2 \omega} E_{l}^{\prime} \exp \left(\overline{i \bar{k}_{l}^{\prime}} \bar{R}\right) \\
E_{l}^{\prime R} \exp \left(i \bar{k}_{l}^{\prime R} \bar{R}\right)=R_{2 \omega} E_{l}^{\prime} \exp \left(i \bar{k}_{l}^{\prime} \bar{R}\right) \\
T_{2 \omega}=\frac{2 n_{2 \omega} \cos \varphi_{2 \omega}^{\prime}}{\cos \varphi_{2 \omega}^{\prime \prime}+n_{2 \omega} \cos \varphi_{2 \omega}^{\prime}} \\
R_{2 \omega}=\frac{n_{2 \omega} \cos \varphi_{2 \omega}^{\prime}-\cos \varphi_{2 \omega}^{\prime \prime}}{n_{2 \omega} \cos \varphi_{2 \omega}^{\prime}+\cos \varphi_{2 \omega}^{\prime \prime}}
\end{gathered}
$$

2) CONDitions AUX Limites POUR L'ONDE ForcÉE. Cette onde ne peut exister que dans la mesure où l'onde de polarisation forcée qui l'induit existe. Lorsque l'onde fondamentale se réfléchit aux limites du deuxième dioptre, elle crée une onde de polarisation qui l'accompagne et le module de l'onde forcée corres- 
pondante $E_{f}^{\prime R}$ est fixé par une relation du type (4a). Sur le dioptre existent donc deux ondes forcées dont les modules et les phases sont bien définis.

Tout comme pour le premier dioptre, les conditions de continuité des composantes tangentielles des champs $\mathrm{E}$ et $\mathrm{H}$ donnent lieu à quatre équations qui imposent l'existence de deux nouvelles ondes libres, l'une réfléchie dans le matériau $\bar{E}_{L}^{\prime R}$, l'autre réfractée dans le vide $\bar{E}_{L}^{\prime \prime}$ (Fig. 5). Ces ondes se propagent dans le plan d'incidence défini par l'onde forcée $\bar{E}_{f}^{\prime}$ qui est aussi le plan de section droite du prisme. De plus, avec la géométrie choisie, toutes ces ondes sont polarisées perpendiculairement au plan d'incidence.

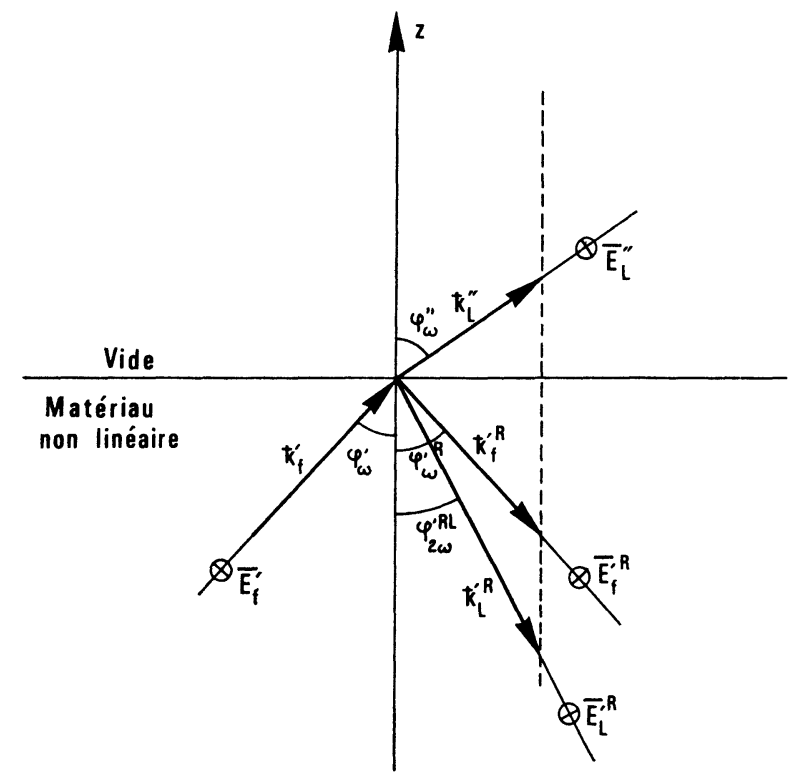

Fig. 5. - Définition des symboles sur le dioptre matériau non linéaire-vide pour l'onde harmonique forcée.

Les notations que nous employons sur la figure 5 sont résumées dans le tableau suivant.

$\begin{array}{lcc}\begin{array}{cc}\text { Onde } \\ -\end{array} & \begin{array}{c}\text { Module } \\ \text { du vecteur d'onde }\end{array} & \begin{array}{c}\text { Angle } \\ \text { avec la normale }\end{array} \\ \bar{E}_{f}^{\prime} & \left|\bar{k}_{f}^{\prime}\right|=n_{\omega} \frac{2 \omega}{c} & \varphi_{\omega}^{\prime} \\ \bar{E}_{f}^{\prime R} & \left|\bar{k}_{f}^{\prime R}\right|=n_{\omega} \frac{2 \omega}{c} & \varphi_{\omega}^{\prime R} \\ \bar{E}_{L}^{R} & \left|k_{L}^{\prime R}\right|=n_{2 \omega} \frac{2 \omega}{c} & \varphi_{\omega}^{\prime R L} \\ \bar{E}_{L}^{\prime \prime} & \left|k_{L}^{\prime \prime}\right|=\frac{2 \omega}{c} & \varphi_{\omega}^{\prime \prime}\end{array}$

Définissons tout d'abord l'onde forcée réfléchie : le coefficient de réflexion de l'onde fondamentale est :

$$
R_{\omega}=\frac{n_{\omega} \cos \varphi_{\omega}^{\prime}-\cos \varphi_{\omega}^{\prime \prime}}{n_{\omega} \cos \varphi_{\omega}^{\prime}+\cos \varphi_{\omega}^{\prime \prime}} .
$$

L'onde forcée réfléchie est liée à l'onde fondamentale réfléchie par :
$E_{f}^{\prime R} \exp \left(\overline{i \bar{k}_{f}^{\prime R}} \bar{R}\right)=4 \pi \frac{\mathrm{d}_{\mathrm{eff}}}{n_{\omega}^{2}-n_{2 \omega}^{2}}\left|E_{\omega}^{\prime R} \exp \left(i{\overline{k_{\omega}^{\prime}}}^{\prime R} \bar{R}\right)\right|^{2}$

avec

$$
E_{\omega}^{\prime R} \exp \left(\overline{i \bar{k}_{\omega}^{\prime}} \bar{R}\right)=R_{\omega} E_{\omega}^{\prime} \exp \left(i \bar{k}_{\omega}^{\prime} \bar{R}\right) .
$$

On peut donc écrire que :

$$
E_{f}^{\prime R} \exp \left(i \bar{k}_{f}^{\prime R} \bar{R}\right)=R_{\omega}^{2} E_{f}^{\prime} \exp \left(i \bar{k}_{f}^{\prime} \bar{R}\right)
$$

La condition aux limites relative au vecteur d'onde donne les relations angulaires suivantes:

$n_{\omega} \sin \varphi_{\omega}^{\prime}=-n_{\omega} \sin \varphi_{\omega}^{\prime R}=-n_{2 \omega} \sin \varphi_{2 \omega}^{\prime R L}=\sin \varphi_{\omega}^{\prime \prime}$.

L'onde libre réfléchie $\bar{E}_{L}^{\prime R}$ a la même direction que l'onde réfléchie par une onde libre à la fréquence $2 \omega$ et dont l'onde réfractée aurait la même direction que l'onde libre dans le vide $\bar{E}_{L}^{\prime \prime}$.

Les conditions relatives à la continuité des champs au point $\mathrm{M}(\overline{\mathrm{OM}}=\bar{R})$ donnent $\bar{E}_{L}^{\prime \prime}$ par :

$$
\begin{gathered}
E_{L}^{\prime \prime} \exp \left(i \bar{k}_{L}^{\prime \prime} \bar{R}\right)=T_{\omega} E_{f}^{\prime} \exp \left(i \bar{k}_{f}^{\prime} \bar{R}\right) \\
T_{\omega}=\frac{\left(1+R_{\omega}^{2}\right) n_{2 \omega} \cos \varphi_{2 \omega}^{\prime R L}+\left(1-R_{\omega}^{2}\right) n_{\omega} \cos \varphi_{\omega}^{\prime}}{\cos \varphi_{\omega}^{\prime \prime}+n_{2 \omega} \cos \varphi_{2 \omega}^{\prime R L}} .
\end{gathered}
$$

REMARQue. - Dans le cas d'une lame à faces parallèles, le vecteur $\overline{\Delta k}$ est aussi perpendiculaire au dioptre de sortie. Les angles $\varphi_{\omega}^{\prime}$ et $\varphi_{2 \omega}^{\prime}$ sont alors tels que

$$
\varphi_{2 \omega}^{\prime \prime}=\varphi_{\omega}^{\prime \prime} \quad \text { et } \quad \varphi_{2 \omega}^{\prime R L}=\varphi_{2 \omega}^{\prime R} .
$$

Les ondes transmises et les ondes libres réfléchies pour les deux composantes de l'onde harmonique ont exactement même direction; on peut alors écrire les conditions aux limites sur le deuxième dioptre simultanément pour ces deux ondes. $\mathrm{Si}$ on néglige la réflexion du fondamental $\left(R_{\omega}=0\right)$ et que l'on additionne les contributions calculées aux deux paragraphes précédents, on retrouve exactement les résultats de Jerphagnon et Kurtz [6].

IV. Interférence de deux ondes de même fréquence. Les expériences d'interférence font intervenir des ondes cohérentes de même fréquence. Ainsi, de même qu'en optique classique, les ondes harmoniques forcées et libres créées dans le matériau interfèrent, mais en optique non linéaire intervient un fait nouveau par rapport aux interférences classiques. En effet, il s'effectue un travail, puisque par l'intermédiaire du matériau, de l'énergie est échangée entre le fondamental et l'harmonique. Nous allons analyser sur un cas simple comment se traduit ce travail.

Le vecteur de Poynting de deux champs à la fré- 
quence $\Omega$, polarisés rectilignement perpendiculairement au plan des vecteurs d'ondes, est :

$\bar{S}_{T}=\frac{c}{8 \pi}\left[n E^{2} \widehat{s}+n^{\prime} E^{\prime 2} \hat{s}^{\prime}+E E^{\prime}\left(\hat{s}+n^{\prime} \hat{s}^{\prime}\right) \cos \Delta \bar{K} \bar{r}\right]$

Les notations employées sont les suivantes (Fig. 6) :

$$
E_{T}=E \hat{e} \exp i(\Omega t-\bar{K} \bar{r})+E^{\prime} \hat{e}^{\prime} \exp i\left(\Omega t-\bar{K}^{\prime} \bar{r}\right)
$$

$\hat{e}=\hat{e}^{\prime} \quad \bar{K}=n \frac{\Omega}{c} \widehat{s} \quad \bar{K}^{\prime}=n^{\prime} \frac{\Omega}{c} \widehat{s}^{\prime} \quad \hat{e} \cdot \hat{s}=\hat{e}^{\prime} \cdot \hat{s}^{\prime}=0$

$$
\overline{\Delta K}=\bar{K}-\bar{K}^{\prime} \text {. }
$$
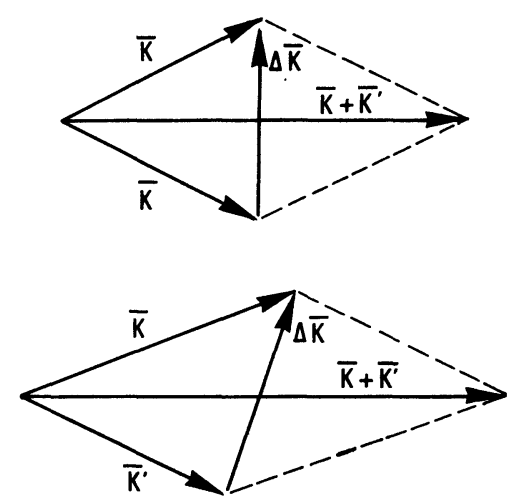

Fig. 6. - Interférences de deux ondes de même fréquence :

a) dans un milieu linéaire où $|\bar{K}|=\left|\bar{K}^{\prime}\right|$

b) dans un milieu non linéaire où $|\bar{K}| \neq\left|\bar{K}^{\prime}\right|$

Les deux premiers termes de (25) sont les vecteurs de Poynting des deux composantes :

$$
\bar{S}=n \frac{c}{8 \pi} E^{2} \hat{s} \quad \bar{S}^{\prime}=n^{\prime} \frac{c}{8 \pi} E^{\prime 2} \hat{s}^{\prime} .
$$

Le terme d'interférence est un vecteur dirigé suivant $\left(\bar{K}+\bar{K}^{\prime}\right)$ mais il est modulé en amplitude en fonction de $\bar{r}$ suivant la direction du vecteur $\left(\bar{K}-\bar{K}^{\prime}\right)$. Le travail fourni par le milieu est proportionnel à :

$\operatorname{div} \bar{S}_{T}=\frac{\Omega}{8 \pi} E E^{\prime}\left(\bar{K}+K^{\prime}\right)\left(\bar{K}-\bar{K}^{\prime}\right) \sin \left(\left(\bar{K}-\bar{K}^{\prime}\right) \bar{r}\right)$.

Dans un milieu linéaire dans lequel on créerait une telle situation, par exemple dans une expérience d'interférence classique, les ondes en interférence seraient telles que $|\bar{K}|=\left|\bar{K}^{\prime}\right|$ et $\left(\bar{K}^{\prime}+\bar{K}\right)$ serait perpendiculaire à $\left(\bar{K}-\bar{K}^{\prime}\right)$, le milieu ne fournirait alors aucun travail.

Dans un milieu non linéaire, en général $|\bar{K}| \neq\left|\bar{K}^{\prime}\right|$, bien que les fréquences soient identiques, et le milieu permet un transfert d'énergie entre le fondamental et l'harmonique. Dans le cas où l'accord de phase est réalisé, $\bar{K}=\bar{K}^{\prime}$ mais l'expression (26) n'est plus correcte, l'onde harmonique ne pouvant pas être décomposée en un nombre fini d'ondes planes.

\section{DEUXIÈME PARTIE}

I. Génération d'harmonique dans un prisme de matériau non linéaire transparent. - Nous allons appliquer la théorie développée dans la première partie au calcul du flux de rayonnement harmonique créé par un pinceau cylindrique d'onde plane, monochromatique, de diamètre $2 W_{0}$ dans un prisme de matériau non linéaire. Une telle situation représente une bonne approximation (voir annexe no 1 ) pour caractériser la configuration de la plupart des expériences de génération de second harmonique (Fig. 1 et 7 ).

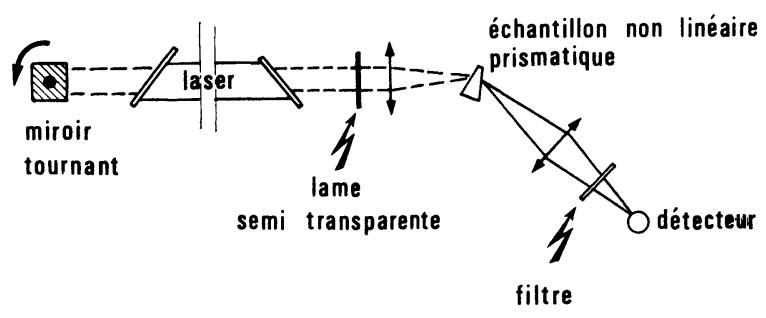

FIG. 7. - Schéma du montage expérimental de génération de second harmonique.

Les faisceaux harmoniques découpent sur la surface du deuxième dioptre trois régions (Fig. 8). Sur la surface $\Sigma_{l}$, seule l'onde $\bar{E}_{l}^{\prime \prime}$ issue de l'onde libre $\bar{E}_{l}^{\prime}$ est

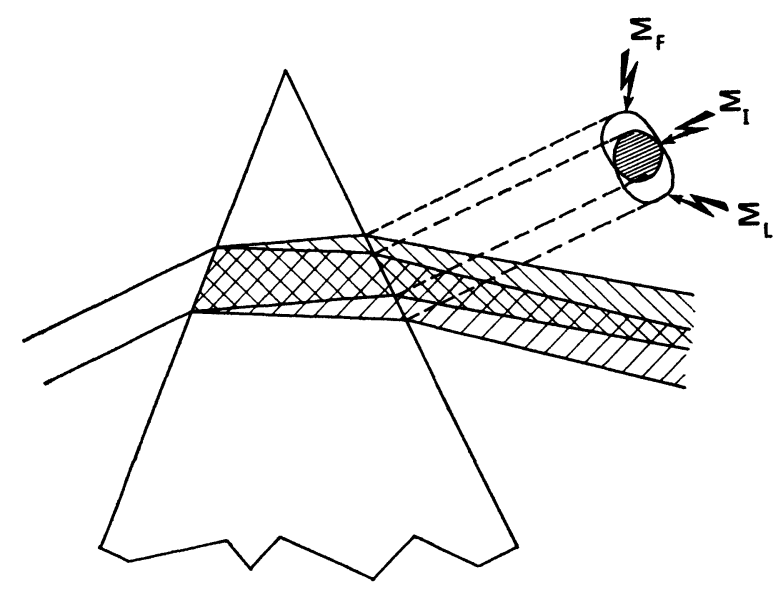

Fig. 8. - Surface découpée sur le second dioptre par les faisceaux harmoniques.

présente ; sur $\Sigma_{L}$, seule arrive l'onde libre $\bar{E}_{L}^{\prime \prime}$ issue de $\bar{E}_{f}^{\prime}$; enfin, sur $\Sigma_{I}$ les deux ondes sont présentes. Sur la surface $\Sigma_{I}$ la présence simultanée de deux ondes cohérentes de même fréquence induit des interférences. Du côté du vide et sur $\Sigma_{I}$, les champs électrique et magnétique s'expriment par les formules suivantes :

$\bar{E}_{T}^{\prime \prime}=\hat{e}_{L}^{\prime \prime} E_{L}^{\prime \prime} \exp \left(i \bar{k}_{L}^{\prime \prime} \bar{R}\right)+\hat{e}_{l}^{\prime \prime} E_{l}^{\prime \prime} \exp \left(i \bar{k}_{l}^{\prime \prime} \bar{R}\right)$

$\left.\bar{H}_{T}^{\prime \prime}=\widehat{(s}_{L}^{\prime \prime} \times \widehat{e}_{L}^{\prime \prime}\right) E_{L}^{\prime \prime} \exp \left(i \bar{k}_{L}^{\prime \prime} \bar{R}\right)$

$$
\left.+\hat{s}_{l}^{\prime \prime} \times \widehat{e}_{l}^{\prime \prime}\right) E_{l}^{\prime \prime} \exp \left(\overline{i k_{l}^{\prime \prime}} \bar{R}\right) .
$$

Le vecteur de Poynting en un point

$$
M(\overline{\mathrm{OM}}=\bar{R}(X, Y, Z))
$$


du dioptre du côté du vide est :

$$
\bar{S}_{T}=\frac{c}{8 \pi} R_{\mathrm{e}}\left[\bar{E}_{T}^{\prime \prime} \times \bar{H}_{T}^{\prime \prime *}\right] .
$$

En utilisant les relations démontrées aux paragraphes précédents, on peut exprimer les champs sur la surface $\Sigma_{I}$ du côté du vide en fonction des champs sur la même surface du côté du diélectrique ; nous définissons le paramètre $D$ par la formule suivante :

$$
E_{f}^{\prime}=D\left|E_{\omega}^{\prime}\right|^{2} .
$$

Pour la configuration retenue :

$$
D=4 \pi \frac{\mathrm{d}_{\mathrm{eff}}}{n_{\omega}^{2}-n_{2 \omega}^{2}}
$$

Finalement le vecteur de Poynting sur $\Sigma_{I}$ prend la forme suivante :

$$
\begin{aligned}
\bar{S}_{T}=\frac{c}{8 \pi}\left(D t_{\omega}^{2} E_{\omega}^{2}\right)^{2}\left(T_{\omega}^{2} \widehat{s}_{L}^{\prime \prime}+A_{l}^{2} T_{2 \omega}^{2} \widehat{s}_{l}^{\prime \prime}+\right. \\
\left.+A_{l} T_{\omega} T_{2 \omega}\left(\hat{s}_{l}^{\prime \prime}+\widehat{s}_{L}^{\prime \prime}\right) \cos (\overline{\Delta k} \bar{R})\right)
\end{aligned}
$$

Le vecteur $\overline{\Delta k}$ défini par la formule (13) est perpendiculaire au premier dioptre (Fig. 9), donc la phase

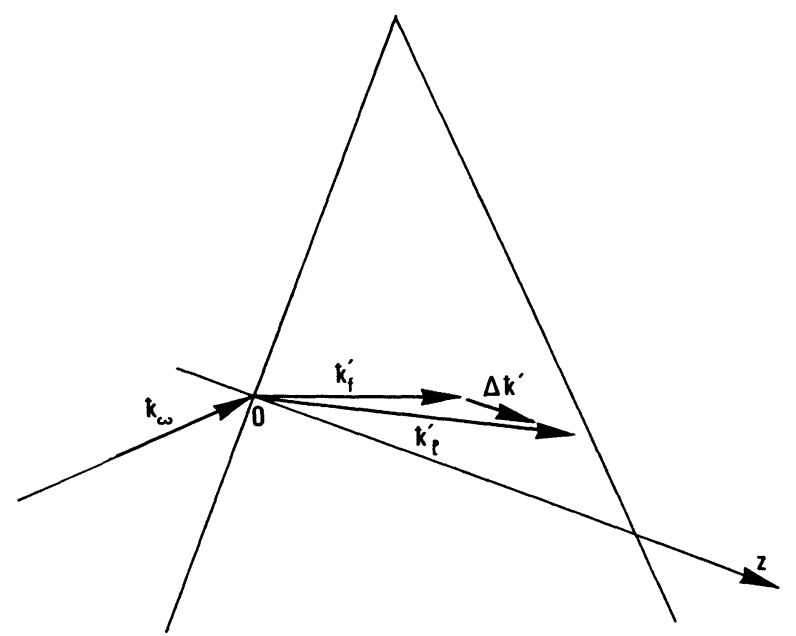

FIG. 9. - Orientation de $\overline{\Delta k^{\prime}}$ par rapport au premier dioptre.

du cosinus ne dépend que de la coordonnée $z$ du point $M$. Cela conduit à introduire la longueur de cohérence pour la direction du faisceau incident $l_{c}(\theta)$.

$$
l_{c}(\theta)=\frac{\lambda_{\omega}}{4\left|\left(n_{2 \omega} \cos \theta_{2 \omega}^{\prime}-n_{\omega} \cos \theta_{\omega}^{\prime}\right)\right|}=\frac{\lambda}{4 \Delta n(\theta)} .
$$

II. Approximations et résultats numériques. - Les grandeurs indicées $2 \omega$ et celles indicées $\omega$ sont en général très voisines. Pour des calculs numériques il est possible de les confondre dans toutes les expressions où elles n'apparaissent pas sous forme de différence. Vérifions ce point sur un exemple numérique.
Soit un matériau d'indice $n=2$ et pour lequel $\Delta n(\theta)=10^{-2}$ alors $\theta_{2 \omega}^{\prime}-\theta_{\omega}^{\prime} \simeq 4^{\prime}$ et si nous posons :

$$
\begin{gathered}
T=T_{2 \omega}=T_{\omega}(1+\varepsilon) \quad A_{l}+1=\eta \\
\cos \varphi^{\prime \prime}=\cos \varphi_{2 \omega}^{\prime \prime}=(1+2 \beta) \cos \varphi_{\omega}^{\prime \prime} .
\end{gathered}
$$

Il vient $: \varepsilon \simeq 10^{-2} \quad \eta \simeq-5 \times 10^{-3} \quad \beta \simeq 10^{-3}$. Pour des échantillons de dimensions usuelles (de l'ordre du centimètre), la séparation des faisceaux est très faible. Les surfaces $\Sigma_{L}$ et $\Sigma_{l}$ sont tout à fait négligeables et la surface $\Sigma_{I}$ est une ellipse d'axes :

$$
2 W_{0} \text { et } 2 W_{0} \frac{\cos \theta^{\prime}}{\cos \theta \cos \varphi^{\prime}} \text {. }
$$

$\mathrm{Vu}$ les dimensions géométriques, il est aisé de concentrer sur le détecteur la totalité de l'énergie à la fréquence $2 \omega$ sortant de l'échantillon. Le signal détecté est alors proportionnel au flux du vecteur de Poynting donné par (30) à travers la surface $\Sigma_{I}$. Le détail du calcul est donné en annexe $\mathrm{n}^{0} \mathbf{3}$ dans le cas le plus général. Nous retiendrons le résultat lorsqu'on se place au minimum de déviation. Les notations utilisées sont précisées à la figure 10. Alors l'ellipse $\Sigma_{I}$ est la

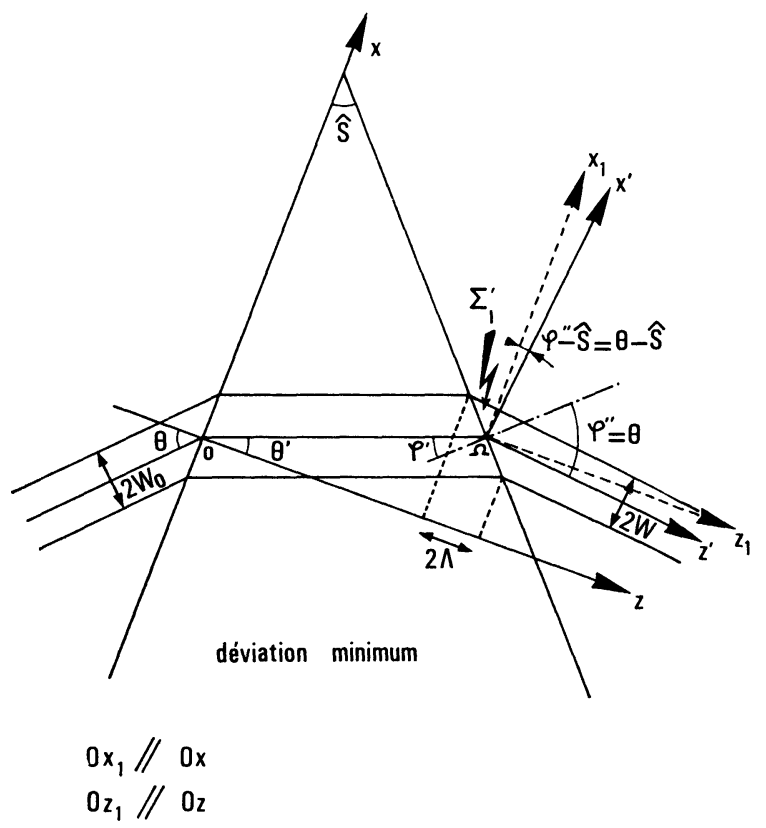

FIG. 10. - Notations utilisées dans le calcul du flux $\Phi_{2 \omega}$ de second harmonique, à la déviation minimum.

section par le deuxième dioptre d'un cylindre de section droite circulaire de diamètre $2 W_{0}$ et dont l'axe est le rayon émergent moyen. On trouve :

$$
\begin{aligned}
\Phi_{2 \omega}=\frac{c}{8 \pi} & \left(D T t_{\omega}^{2} E_{\omega}^{2}\right)^{2} 2 \pi W_{0}^{2} \\
& \times\left(1-F\left(\frac{\pi \Lambda}{l_{c}(\theta)}\right) \cos \left(\frac{\pi L}{l_{c}(\theta)}\right)\right)
\end{aligned}
$$

$L$ est la cote du centre $\Omega$ de $\Sigma_{I}$, 
$\Lambda=W_{0} \sin S / \cos \varphi^{\prime \prime}$ est la projection du demi-grand axe de $\Sigma_{I}$ sur oz ( $2 \Lambda$ représente donc la variation de la coordonnée $z$ sur l'extension du faisceau), $F(t)=2 J_{1}(t) / t$ est une fonction auxilliaire (définie en annexe $n^{\circ} 3$ ) de la fonction de Bessel $J_{1}$ d'ordre un.

Dans le cas où le prisme n'est pas au minimum de déviation, la formule (33) conserve sa forme, seule l'expression de $\Lambda$ (dont la signification physique reste la même) est plus compliquée ; il s'introduit également en facteur un terme tenant compte de l'écart par rapport au minimum de déviation.

Les extremums de la fonction $F$ sont donnés en annexe, ainsi que sa courbe représentative. Son module est toujours inférieur ou égal à l'unité, ce qui montre que lorsque $L$ varie, les minimums de $\Phi_{2 \omega}$ ne sont pas nuls et que l'amplitude du signal dépend non seulement des propriétés non linéaires du matériau et de la densité de puissance du fondamental, mais aussi de la géométrie de l'échantillon, en particulier de l'angle au sommet du prisme et de l'extension du faisceau.

La fonction $J_{1}(t)$ est équivalente à $\frac{1}{2} t$ quand $t$ tend vers zéro, par conséquent, $F(t)$ tend vers l'unité. Pour une lame à faces parallèles où $\Lambda=0, F(0)=1$. Le flux est alors proportionnel à $\sin ^{2}\left(\pi L / 2 l_{c}\right)$ et l'on retrouve l'analyse de l'expérience classique de Maker [7] fait par Jerphagnon et Kurtz [6].

Remarquons qu'un choix judicieux des paramètres permet de détruire complètement la partie oscillante de $\Phi_{2 \omega}$. Pour cela, il suffit de choisir $W_{0}$ et $S$ de manière à ce que $\pi \Lambda / l_{c}$ soit un zéro de la fonction de Bessel $J_{1}$. On peut encore avoir un signal constant lorsque la longueur de cohérence $l_{c}(\theta)$ est très faible devant $\Lambda$, en effet $|F(t)|$ est inférieur à $3 \%$ dès que $t$ est supérieur à 10. Cela peut donner une nouvelle méthode de mesure de susceptibilité non linéaire par comparaison directe, pour autant que l'on connaisse les indices du matériau. A la fin de cet article, nous présentons les courbes $\Phi_{2 \omega}(L)$ correspondant à un certain nombre de configurations (Fig. 12 à 17).

III. Milieux absorbants. - Pour décrire l'absorption d'un matériau tout en conservant formellement la structure des lois de l'optique, il est commode d'introduire un indice complexe $\stackrel{0}{n}=n(1+i \kappa)$ de sorte que le vecteur d'onde $\frac{0}{k}=\frac{\Omega}{\mathrm{c}} n(1+i \kappa) \hat{s}$ ait une partie imaginaire qui décrira l'atténuation exponentielle dans le matériau.

Nous rappelons, dans l'annexe no 4, un certain nombre de résultats classiques concernant les matériaux absorbants. Les points essentiels à retenir sont les suivants. Les modes propres de propagation du milieu sont des ondes de polarisation elliptique. La traversée d'un dioptre s'accompagne d'une variation simultanée de module et de phase (les facteurs de Fresnel sont complexes).

Dans les expériences d'optique non linéaire en transmission, les matériaux fortement absorbants pour les fréquences fondamentale et harmonique ne présentent pas l'intérêt pratique.

Seuls sont à retenir les matériaux transparents, voire légèrement absorbants, ou les matériaux ayant une absorption sélective sur une des fréquences et ce pour des raisons particulières que nous examinerons plus loin.

Cette restriction dans le choix des matériaux apporte une grande simplification du formalisme décrivant l'optique de ces corps.

Considérons un exemple pour fixer les ordres de grandeur : le coefficient d'absorption est défini par $I=I_{0} \exp (-a x)$. Pour un champ

$$
E=E_{0} \exp \left[i \frac{\Omega}{c} \stackrel{0}{n} x\right]
$$

il vient :

$$
a=2 \frac{\Omega}{c} n \kappa=4 \frac{\pi n}{\lambda} \kappa
$$

pour $\lambda=10 \mu$ et un matériau d'indice $n=2$ ayant un coefficient d'absorption relativement élevé $a=10 \mathrm{~cm}^{-1}$, alors $\kappa=4 \times 10^{-4}$.

On conçoit que pour $a<10 \mathrm{~cm}^{-1}$ on puisse dans tout le domaine optique négliger à coup sûr les puissances de $\kappa$ et même $\kappa$ lui-même lorsqu'il n'apparaît pas dans les fonctions dont la variation dépend de manière critique de ses valeurs (essentiellement des exponentielles décroissantes).

On pourra s'assurer d'après les résultats de l'annexe no 4 que pour de telles valeurs de $\kappa$ l'ellipticité des modes propres et les variations de phase accompagnant la traversée d'un dioptre sont effectivement négligeables et on admettra que l'on conserve les lois de l'optique des milieux transparents en dehors de l'atténuation exponentielle des ondes planes.

Nous allons dans ce chapitre étudier la génération $d$ 'harmonique dans les milieux légèrement absorbants et au voisinage d'une bande d'absorption. Nous écrirons ainsi que :

$$
\bar{E}_{T}(2 \omega)=\hat{e}_{l}^{\prime} E_{l}^{\prime} \exp \left(\overline{i \bar{k}_{l}^{\prime}} \bar{r}\right)+\hat{e}_{f}^{\prime} E_{f}^{\prime} \exp \left(i \bar{k}_{f}^{\prime} \bar{r}\right) .
$$

Les polarisations et les amplitudes des deux composantes peuvent dans tout les cas se calculer à partir des caractéristiques linéaires et non linéaires du matériau. Nous utiliserons les notations suivantes, simples extensions des notations des paragraphes précédents.

$$
\begin{gathered}
\bar{k}_{f}^{\prime}=2 \bar{k}_{\omega}^{\prime}=\frac{2 \omega}{c}{\stackrel{0}{n_{\omega}}}^{\hat{s}_{f}^{\prime}}=\frac{2 \omega}{c} n_{\omega}\left(1+i \kappa_{\omega}\right) \hat{s}_{f}^{\prime} \\
a_{\omega}=\frac{2 \omega}{c} n_{\omega} \kappa_{\omega} \\
\bar{k}_{l}^{\prime}=\frac{2 \omega}{c} \stackrel{0}{n}_{2 \omega} \hat{s}_{l}^{\prime}=\frac{2 \omega}{c} n_{2 \omega}\left(1+i \kappa_{2 \omega}\right) \hat{s}_{l}^{\prime} \\
a_{2 \omega}=\frac{4 \omega}{c} n_{2 \omega} \kappa_{2 \omega} .
\end{gathered}
$$


Les grandeurs $\bar{k}_{f}^{\prime}, \hat{s}_{f}^{\prime}, \bar{k}_{l}^{\prime}, \hat{s}_{l}^{\prime}$ sont des vecteurs complexes. Leurs principales propriétés sont rappelées en annexe no 4.

1) GÉNÉRATION D'HARMONIQUE DANS UN MATÉRIAU PEU ABSORBANT. - Pour de tels matériaux, $\kappa$ est très petit devant l'unité. On peut montrer (voir annexe $n^{\circ}$ 4) que les lois de l'optique des matériaux transparents sont applicables au premier ordre; la seule modification appréciable par rapport aux corps transparents est l'atténuation exponentielle de l'amplitude d'une onde. Le facteur de phase $\left(\overline{i k^{\prime}} \bar{r}\right)$ s'écrit alors : $i \bar{k}^{\prime} \bar{r}=\frac{2 \omega}{c} i\left(n \sin \theta^{\prime} x+n \cos \theta^{\prime} z\right)-\frac{2 \omega}{c} \frac{n \kappa}{\cos \theta^{\prime}} z$

Nous utiliserons donc sans modification les résultats du chapitre précédent concernant les rapports d'amplitude et de phase des deux composantes du champ harmonique lors de la traversée des dioptres, mais nous ferons intervenir la décroissance exponentielle en fonction de la profondeur de pénétration.

La formule (30) donnant l'expression du vecteur de Poynting sur la surface $\Sigma_{I}$ se généralise ; comme les vecteurs d'onde sont complexes, il apparaît de nouveaux termes, ainsi :

$$
\begin{aligned}
& i\left(\bar{k}_{\omega}^{\prime} \bar{r}-\bar{k}_{\omega}^{\prime *} \bar{r}\right)=-\frac{4 \omega}{c} \frac{n_{\omega}}{\cos \theta_{\omega}^{\prime}} z=-2 \frac{\kappa_{\omega}}{\cos \theta_{\omega}^{\prime}} z=-2 \gamma_{\omega} z \\
& i\left(\bar{k}_{2 \omega}^{\prime} \bar{r}-\bar{k}_{2 \omega}^{\prime *} \bar{r}\right)=-\frac{4 \omega}{c} \frac{n_{2 \omega} \kappa_{2 \omega}}{\cos \theta_{2 \omega}^{\prime}} z=-\frac{a_{2 \omega}}{\cos \theta_{2 \omega}} z=-\gamma_{2 \omega} z \\
& i\left(\bar{k}_{2 \omega}^{\prime} \bar{r}-\bar{k}_{\omega}^{\prime *} \bar{r}\right)=\frac{2 \omega}{c} i\left(n_{2 \omega} \cos \theta_{2 \omega}^{\prime}-n_{\omega} \cos \theta_{\omega}^{\prime}\right) z-\frac{2 \omega}{c}\left(\frac{n_{\omega} \kappa_{\omega}}{\cos \theta_{\omega}^{\prime}}+\frac{n_{2 \omega} \kappa_{2 \omega}}{\cos \theta_{2 \omega}^{\prime}}\right)=i \frac{\pi}{l_{c}(\theta)} z-\left(\gamma_{\omega}+\frac{1}{2} \gamma_{2 \omega}\right) z
\end{aligned}
$$

La formule équivalente à la formule (30) est la suivante :

$$
\begin{array}{r}
S_{T}=\frac{c}{8 \pi}\left(D t_{\omega}^{2} E_{\omega}^{2}\right)^{2}\left(\hat{s}_{L}^{\prime \prime} T_{\omega}^{2} \exp \left(-2 \gamma_{\omega} z\right)+\hat{s}_{l}^{\prime \prime} A_{l}^{2} T_{2 \omega}^{2} \exp \left(-\gamma_{2 \omega} z\right)\right. \\
\left.+\left(\hat{s}_{l}^{\prime \prime}+\hat{s}_{L}^{\prime \prime}\right) T_{\omega} T_{2 \omega} A_{l} \exp \left(-\left(\gamma_{\omega}+\frac{1}{2} \gamma_{2 \omega}\right) z\right) \cos \left(\frac{\pi z}{l_{c}(\theta)}\right)\right)
\end{array}
$$

Le calcul du flux se mène de la même manière que pour les milieux transparents. Là encore, nous confondons les variables indicées $2 \omega$ et celles indicées $\omega$ lorsque leurs valeurs sont très proches (Nota. Ce n'est pas le cas pour les coefficients d'absorption qui peuvent être très différents.) Le calcul est donné en annexe $n^{0} 3$; les formules générales y sont données.

Le flux présente toujours en fonction de l'épaisseur de matériau traversée une série de minimums et de maximums dont la distance est $l_{c}(\theta)$ mais dont l'amplitude a une décroissance à allure exponentielle. En pratique on pourra utiliser la formule suivante:

$$
\begin{gathered}
\Phi_{2 \omega}=\frac{c}{8 \pi}\left(D t_{\omega}^{2} T E_{\omega}^{2}\right)^{2} \pi W_{0}^{2} \\
\times\left[\exp \left(-\gamma_{2 \omega} L\right)+\exp \left(-2 \gamma_{\omega} L\right)-\right. \\
\left.-2 \exp \left(-\left(\gamma_{\omega}+\frac{1}{2} \gamma_{2 \omega}\right) L\right) \cos \frac{\pi L}{l_{c}(\theta)} F\left(\frac{\pi \Lambda}{l_{c}(\theta)}\right)\right]
\end{gathered}
$$

qui est valable au minimum de déviation et lorsque les coefficients d'absorption sont suffisamment faibles pour que $\gamma L \ll 1$ et $\gamma l_{c} / \pi \ll 1$. Des exemples de courbes $\Phi_{2 \omega}(L)$ sont donnés dans les figures 16 et 17 .

2) GÉNÉRATION D'HARMONIQUE AU VOISINAGE D'UNE BANDE d'ABSORPTION. - L'examen de la formule (42) montre que même si l'absorption est importante pour l'une des deux fréquences et si elle l'est beaucoup moins pour l'autre, le second harmonique peut encore être observé.

Si par exemple $a_{\omega} \simeq 0$ et $a_{2 \omega}$ est suffisamment grand, pour une épaisseur de matériau traversé supérieure à $1 / a_{2 \omega}$, le vecteur de Poynting devient égal à :

$$
S_{T}=\frac{c}{8 \pi}\left(D t_{\omega}^{2} E_{\omega}^{2}\right)^{2} T_{\omega}^{2} \hat{s}_{L}^{\prime \prime}
$$

La première conséquence de cette absorption préférentielle est la disparition du terme oscillant en fonction de l'épaisseur. On continue cependant à observer du second harmonique là où l'onde fondamentale est présente.

Pour savoir quelle est l'intensité de cet harmonique, il faut dans la formule ci-dessus expliciter le terme $D^{2}$. Nous supposerons toujours avoir un matériau de symétrie ponctuelle $\overline{43} \mathrm{~m}$. Soient alors $\stackrel{0}{\chi}_{\omega}$ et $\stackrel{0}{\chi}_{2 \omega}$ les 
seuls éléments non nuls du tenseur de susceptibilité $\mathrm{du}$ premier ordre du matériau. On a :

$$
\begin{aligned}
& \varepsilon_{\omega}=\stackrel{0}{n}_{\omega}^{2} I=\left(1+4 \pi \tilde{0}_{\omega}\right) I ; \\
& \varepsilon_{2 \omega}={\stackrel{0}{n_{2 \omega}^{2}}}_{2}=\left(1+4 \pi \stackrel{0}{\chi}_{2 \omega}\right) I
\end{aligned}
$$

où $I$ est le tenseur unité.

(Les susceptibilités linéaires sont des grandeurs complexes pour ces matériaux, c'est leur partie imaginaire qui décrit justement l'absorption.)

L'extension des résultats du premier paragraphe de la première partie au cas où les indices sont complexes montre que :

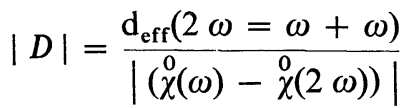

où l'on a fait apparaître la dépendance en fréquence de $\mathrm{d}_{\text {eff }}$ et $\chi$. Dans l'expression des susceptibilités du deuxième ordre et de celles du premier ordre apparaissent des dénominateurs de la forme $\left(\omega_{n 0}-\Omega-i \Gamma_{n}\right)$ où $\omega_{n 0}=\omega_{n}-\omega_{0}$ (différence des fréquences correspondant à l'état de base 0 et à l'état excité $n$ du système), $\Gamma_{n}$ est lié à la durée de vie de ce même niveau et $\boldsymbol{\Omega}=\omega$ ou $2 \omega$ suivant la susceptibilité considérée. Il s'en suit une compétition entre les deux processus d'absorption (augmentation des susceptibilités linéaires) et de création d'harmonique (augmentation des susceptibilités non linéaires). L'étude détaillée de cette compétition doit se mener sur chaque cas particulier en tenant compte des éléments de matrice qui interviennent dans les expressions des susceptibilités.

Miller, Kleinman et Savage [10] ont étudié expérimentalement ce phénomène, dans le sulfure de cadmium en utilisant pour source un laser à néodyme $\left(\lambda_{\omega}=1,06 \mu\right)$; ils ont observé une intensité harmonique à peu près constante en fonction de l'absorption (la variation de celle-ci était obtenue par changement de température). L'analyse de cette expérience a été considérée par Afans'ev et Manykin [11]. Plus récemment, Soref et Moos [12] ont repris et expliqué des expériences de ce type sur des alliages $\mathrm{ZnS}-\mathrm{CdS}$ et CdS-CdSe dont l'absorption pouvait être contrôlée par variation de la composition.

IV. Matériaux biréfringents. - L'extension des résultats des chapitres précédents au cas de matériaux biréfringents demande certaines précautions. Pour de tels corps, à une onde fondamentale incidente correspondent deux ondes réfractées de propagation différente et de polarisations orthogonales. Ces deux ondes, compte tenu des symétries du tenseur de susceptibilité $\mathrm{du}$ deuxième ordre, induisent un certain nombre d'ondes forcées à la fréquence double, qui interfèrent entre elles et avec les deux ondes libres à la fréquence harmonique qui se propagent dans le matériau.

Dans le cas le plus général, huit ondes à la fréquence harmonique peuvent coexister dans le milieu ( 3 ondes forcées ordinaires et 3 ondes forcées extraordinaires de vecteurs d'onde $2 \bar{k}_{e}^{\prime}(\omega), 2 \bar{k}_{o}^{\prime}(\omega),\left(k_{e}^{\prime}(\omega)+k_{o}^{\prime}(\omega)\right)$, et
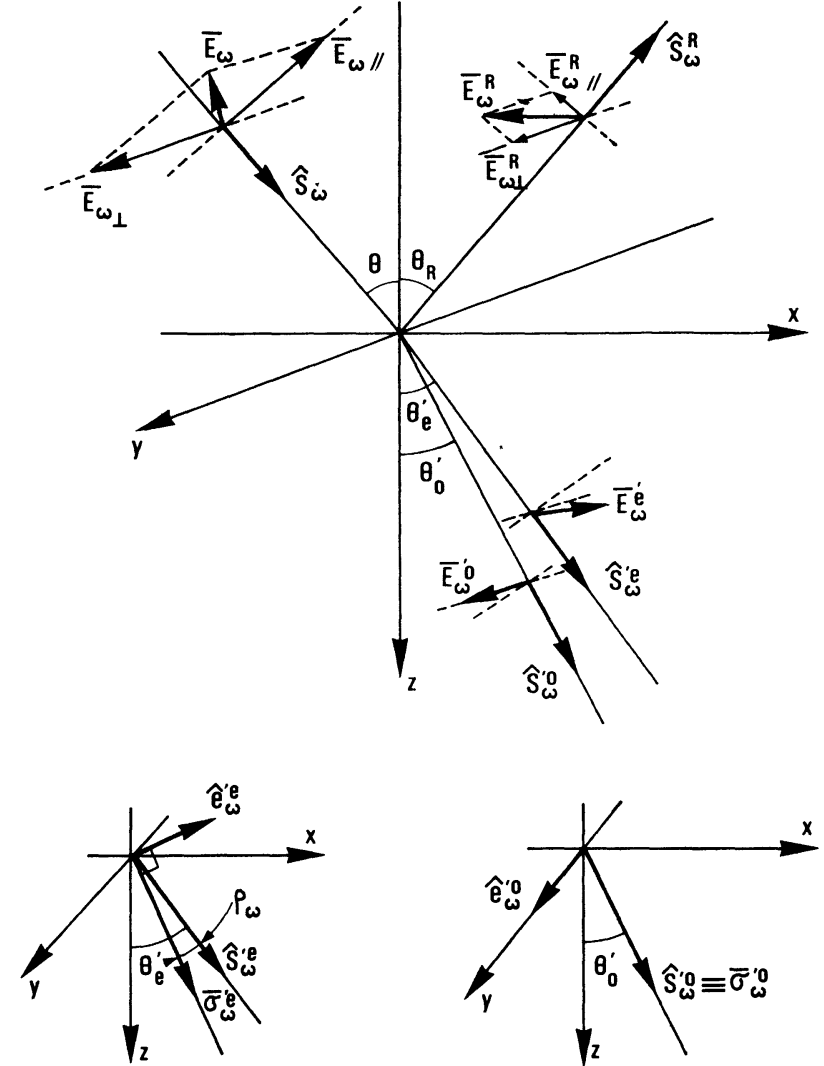

Fig. 11. - Cas des matériaux biréfringents : $a$. - traversée $\mathrm{du}$ dioptre pour l'onde fondamentale ; $b$. - direction de propagation de l'énergie pour l'onde fondamentale extraordinaire ; c. - direction de propagation de l'énergie pour l'onde fondamentale ordinaire.

deux ondes libres de vecteurs d'onde $\bar{k}_{e}^{\prime}(2 \omega)$ et $\bar{k}_{o}^{\prime}(2 \omega)$. Les interférences peuvent donc être très compliquées et dépendent de manière critique de la configuration particulière choisie.

Comme il est impossible de traiter dans toute sa généralité la génération d'harmonique c'ans un matériau biréfringent, nous analyserons un exemple précis. Considérons un corps de symétrie ponctuelle 32 (quartz, cinabre, tellure); le premier dioptre étant choisi de manière que les axes oxyz précédemment définis soient les axes cristallins, oz est l'axe optique. L'onde fondamentale de polarisation quelconque donne naissance à une onde ordinaire et à une onde extraordinaire (Fig. 11) pour lesquelles les facteurs de transmission sont les suivants :

$$
\begin{gathered}
t_{/ /}=\frac{2 \cos \theta}{n_{\omega}^{e} \cos \theta+\cos \theta_{\omega}^{\prime e}} \quad t_{\perp}=\frac{2 \cos \theta}{n_{\omega}^{o} \cos \theta_{\omega}^{\prime o}+\cos \theta} \\
\sin \theta=n_{\omega}^{o} \sin \theta_{\omega}^{\prime o}=n_{\omega}^{e}\left(\theta^{\prime}\right) \sin \theta_{\omega}^{\prime e} .
\end{gathered}
$$

Le champ à la fréquence $\omega$ dans le milieu est donc :

$$
\begin{aligned}
\bar{E}_{\omega}^{\prime}=\hat{e}_{\omega}^{\prime o} E_{o}^{\prime} & \exp \left(i \frac{\omega}{c} n_{\omega}^{o} \hat{s}_{\omega}^{\prime o} \bar{r}\right) \\
& +\hat{e}_{\omega}^{\prime e} E_{e}^{\prime} \exp \left(i \frac{\omega}{c} n_{\omega}^{e}\left(\theta_{\omega}^{\prime e}\right) \hat{s}_{\omega}^{\prime e} \bar{r}\right) .
\end{aligned}
$$


Pour l'onde ordinaire, $\hat{e}_{\omega}^{\prime o}$ est parallèle à oy et la phase et l'énergie ont même direction de propagation. Pour l'onde extraordinaire, la phase se propage suivant $\hat{s}_{\omega}^{\prime e}$ mais l'énergie se propage suivant

$$
\bar{\sigma}_{\omega}^{\prime e}=\hat{e}_{\omega}^{\prime e} \times\left(\hat{s}_{\omega}^{\prime e} \times \hat{e}_{\omega}^{\prime e}\right)
$$

qui fait un angle $\rho_{\omega}$ avec $\hat{s}_{\omega}^{\prime e}$ (voir annexe $\mathrm{n}^{\circ} 5$ ), de sorte que :

$$
\hat{e}_{\omega}^{\prime e}=\hat{x} \cos \left(\theta_{\omega}^{\prime e}+\rho_{\omega}\right)-\hat{z} \sin \left(\theta_{\omega}^{\prime e}+\rho_{\omega}\right)
$$

(Fig. 11).
Pour les corps de symétrie ponctuelle 32 et en supposant que les relations de Kleinman sont vérifiées, le tenseur de susceptibilité du deuxième ordre a trois éléments non nuls et la polarisation non linéaire s'exprime de la manière suivante :

$$
\begin{aligned}
& P_{x}(2 \omega)=d_{11}\left(E_{x}^{\prime 2}(\omega)-E_{y}^{\prime 2}(\omega)\right) \\
& P_{y}(2 \omega)=-2 d_{11} E_{x}^{\prime}(\omega) E_{y}^{\prime}(\omega) . \\
& P_{z}=0
\end{aligned}
$$

Compte tenu de (48), la polarisation dans le matériau a trois composantes :

$$
\begin{aligned}
& P_{x}^{o}=-d_{11} E_{y}^{\prime 2}(\omega)=-d_{11} E_{o}^{\prime 2} \exp \left(i \frac{2 \omega}{c} n_{\omega}^{o} \hat{s}_{\omega}^{\prime o} \bar{r}\right) \\
& P_{x}^{e}=d_{11} E_{x}^{\prime 2}(\omega)=d_{11} E_{e}^{\prime 2} \cos ^{2}\left(\theta^{e}+\rho_{\omega}\right) \exp \left(i \frac{2 \omega}{c} n_{\omega}^{e}\left(\theta_{\omega}^{\prime e}\right) \hat{s}_{\omega}^{\prime e} \bar{r}\right) \\
& P_{y}^{e o}=-2 d_{11} E_{x}^{\prime}(\omega) E_{y}^{\prime}(\omega)=-2 d_{11} E_{e}^{\prime} E_{o}^{\prime} \cos \left(\theta_{\omega}^{\prime e}+\rho_{\omega}\right) \exp \left(i \frac{2 \omega}{c} n_{\omega}^{e o}\left(\theta_{\omega}^{\prime e}\right) \hat{s}_{\omega}^{\prime e o} \bar{r}\right)
\end{aligned}
$$

Nous avons posé :

$$
\begin{aligned}
\bar{k}_{e}^{\prime}(\omega)+\bar{k}_{o}^{\prime}(\omega) & =\frac{\omega}{c}\left(n_{\omega}^{e}\left(\theta_{\omega}^{\prime e}\right) \hat{s}_{\omega}^{\prime e}+n_{\omega}^{o} \hat{s}_{\omega}^{\prime o}\right) \\
& =\frac{2 \omega}{c} n_{\omega}^{e o}\left(\theta_{\omega}^{\prime e}\right) s_{\omega}^{\prime e}
\end{aligned}
$$

Chacune de ces sources induit dans le cristal une onde forcée de même phase solution d'une équation inhomogène semblable à l'équation (2). Dans l'annexe $\mathrm{n}^{\circ} 2$ nous donnons la méthode de calcul des solutions particulières de l'équation inhomogène avec source constante ainsi que les résultats pour les matériaux biréfringents dans les deux cas envisagés ici. Ces calculs montrent que les trois ondes forcées s'expriment de la manière suivante en fonction de l'onde fondamentale.

- Onde extraordinaire forcée d'indice $n_{\omega}^{e}\left(\theta_{\omega}^{\prime e}\right)=n_{1}$ :

$$
\hat{a}_{1}^{\prime} A_{1}^{\prime}=-\frac{4 \pi d_{11}}{\left(n_{2 \omega}^{e}\left(\theta_{\omega}^{\prime e}\right)\right)^{2}-\left(n_{\omega}^{e}\left(\theta_{\omega}^{\prime e}\right)\right)^{2}}\left(\frac{n_{2 \omega}^{e}\left(\theta_{\omega}^{\prime e}\right)}{n_{2 \omega}^{o}}\right)^{2} E_{e}^{\prime 2} \cos ^{2}\left(\theta_{\omega}^{\prime e}+\rho_{\omega}\right)\left(\hat{x}-\left(\frac{n_{\omega}^{e}\left(\theta_{\omega}^{\prime e}\right)}{n_{2 \omega}^{e}}\right)(\hat{s} \hat{x}) \hat{s}\right) .
$$

- Onde extraordinaire forcée d'indice $n_{\omega}^{0}=n_{2}$ :

$$
\hat{a}_{2}^{\prime} A_{2}^{\prime}=\frac{4 \pi d_{11}}{\left(n_{2 \omega}^{e}\left(\theta_{\omega}^{\prime o}\right)\right)^{2}-\left(n_{\omega}^{o}\right)^{2}}\left(\frac{n_{2 \omega}^{e}\left(\theta_{\omega}^{\prime o}\right)}{n_{2 \omega}^{o}}\right)^{2} E_{o}^{\prime 2}\left(\hat{x}-\left(\frac{n_{\omega}^{o}}{n_{2 \omega}^{e}}\right)^{2} \hat{(s \hat{x})} \hat{s}\right) .
$$

- Onde ordinaire forcée d'indice $n_{\omega}^{e 0}\left(\theta_{\omega}^{\prime e}\right)=n_{3}$ :

$$
\hat{a}_{3}^{\prime} A_{3}^{\prime}=\frac{8 \pi d_{11}}{\left(n_{2 \omega}^{o}\right)^{2}-\left(n_{\omega}^{e e}\left(\theta_{\omega}^{\prime e}\right)\right)^{2}} E_{e}^{\prime} E_{o}^{\prime} \cos \left(\theta_{\omega}^{\prime e}+\rho_{\omega}\right) \hat{y} .
$$

- Onde extraordinaire libre d'indice

$$
n_{2 \omega}^{e}\left(\theta_{2 \omega}^{\prime e}\right)=n_{4}: \quad \hat{a}_{4}^{\prime} A_{4}^{\prime} .
$$

- Onde ordinaire libre d'indice

$$
n_{2 \omega}^{o}=n_{5}: \quad \hat{a}_{5}^{\prime} A_{5}^{\prime} .
$$

Les amplitudes des deux ondes libres sont déterminées par les conditions aux limites (voir annexe $n^{\circ} 5$ ).

Nous avons introduit les notations $\hat{a}_{i}^{\prime} A_{i}^{\prime}$ pour des commodités d'écriture. Si on pose que $\Phi_{i}=n_{i} \frac{2 \omega}{c} \hat{s}_{i}^{\prime} \bar{r}$, le vecteur de Poynting à la fréquence harmonique est :

$$
\begin{aligned}
S_{2 \omega}= & \frac{c}{8 \pi} R_{e} \\
& \times\left(\sum_{i, j} n_{j} \hat{a}_{i}^{\prime} \times\left(\hat{s}_{j}^{\prime} \times \hat{a}_{j}^{\prime}\right) A_{i} A_{j} \exp \left(i\left(\Phi_{i}-\Phi_{j}\right)\right)\right) .
\end{aligned}
$$

Les interférences sont très complexes; le flux du vecteur de Poynting est la somme d'une série de fonctions à variations sinusoïdales, dont la période est liée à une longueur de cohérence du type $l_{i j}=\lambda / 4\left(n_{i}-n_{j}\right)$.

Dans la pratique, ce type de matériaux offre un très grand nombre de possibilités. L'utilisation d'un faisceau polarisé permet d'isoler un certain nombre de composantes, par exemple si dans le développement 


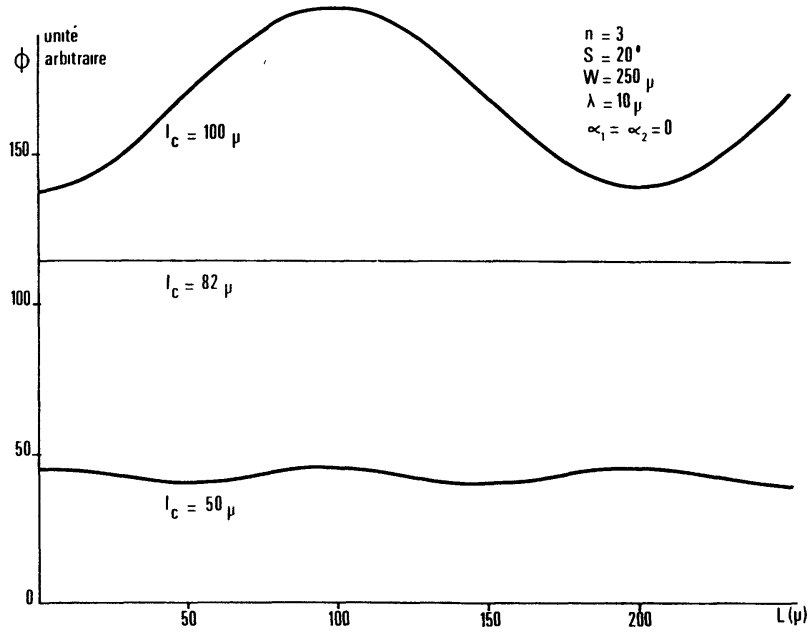

Fig. 12. - Courbes représentatives de la fonction $\Phi_{2 \omega}(L)$ pour différentes valeurs de la longueur de cohérence.

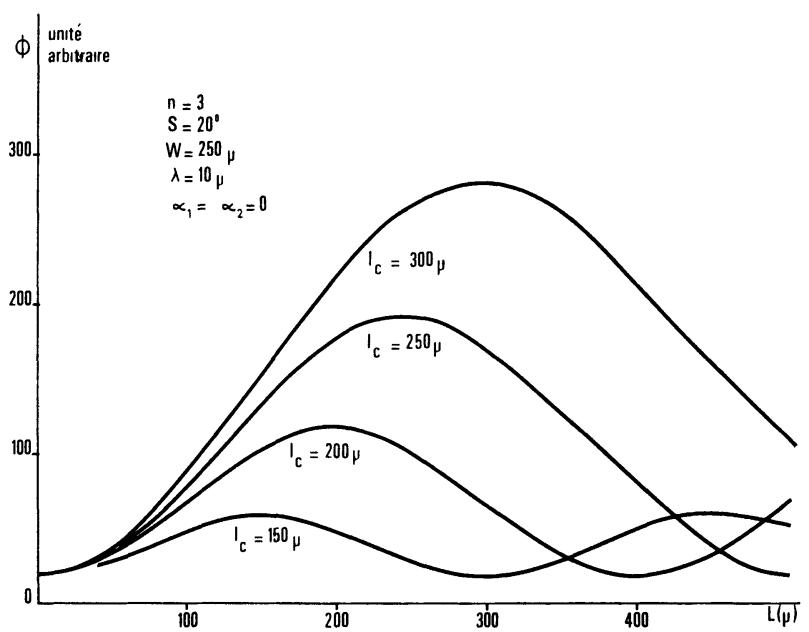

Fig. 13. - Courbes représentatives de la fonction $\Phi_{2 \omega}(L)$ pour différentes valeurs de la longueur de cohérence.

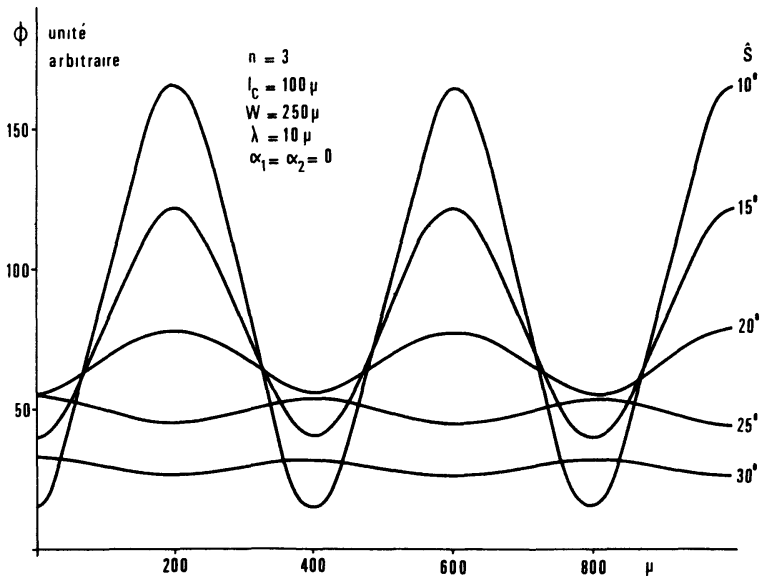

FIG. 14. - Courbes représentatives de la fonction $\Phi_{2 \omega}(L)$ pour différentes valeurs de l'angle du prisme.

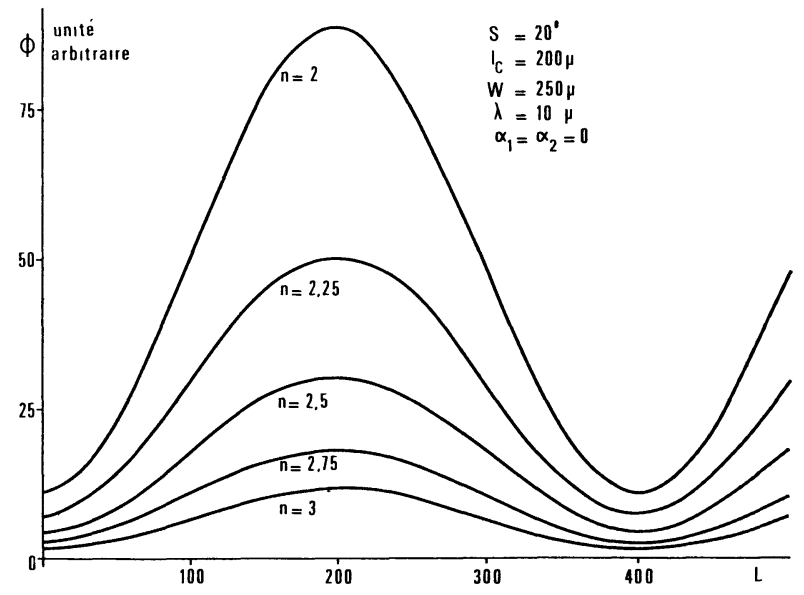

Fig. 15. - Courbes représentatives de la fonction $\Phi_{2 \omega}(L)$ pour différentes valeurs de l'indice du matériau.

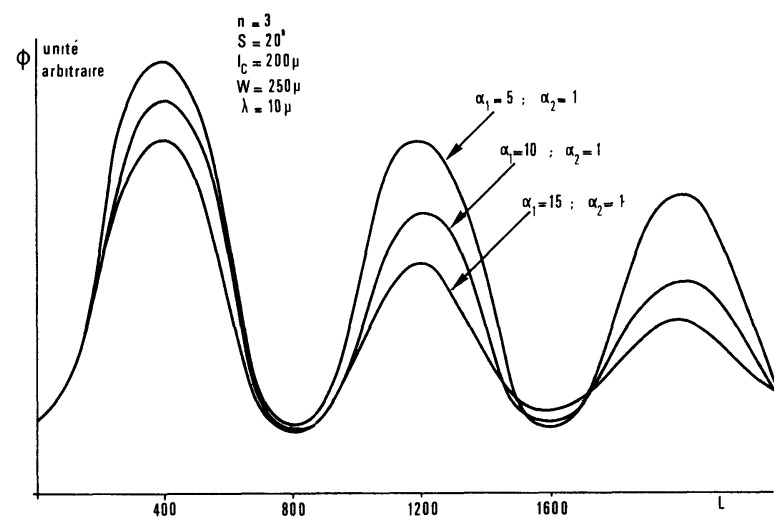

Fig. 16. - Courbes représentatives de la fonction $\Phi_{2_{\omega}}(L)$ pour différentes valeurs des coefficients d'absorption.

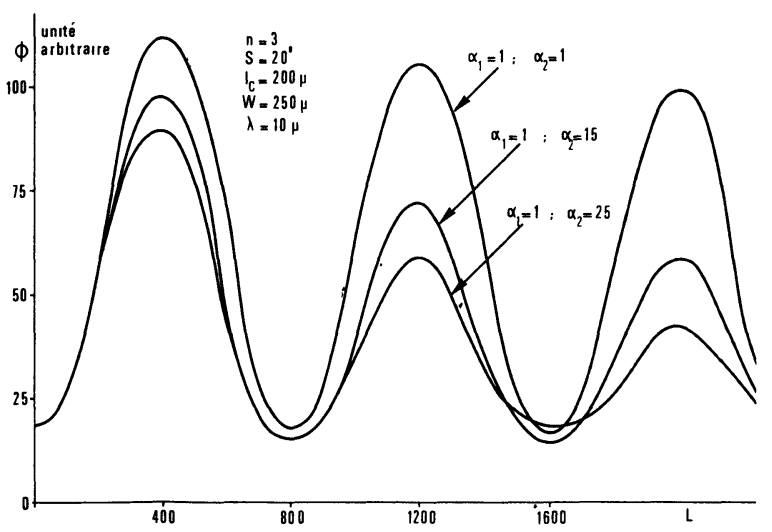

FIG. 17. - Courbes représentatives de la fonction $\Phi_{2 \omega}(L)$ pour différentes valeurs des coefficients d'absorption

ci-dessus $E_{\omega}^{\prime e}=0$, seules les ondes $\widehat{a}_{2}^{\prime} A_{2}$ et $\widehat{a}_{4}^{\prime} A_{4}$ subsistent et pour leurs interférences, la théorie du chapitre 1 , deuxième partie, s'applique sans modification. On peut au contraire utiliser une onde fondamentale de polarisation connue mais ayant une composante ordinaire et une composante extraordinaire et retirer des franges compliquées observées des renseignements sur les signes des éléments du tenseur de 
susceptibilité, ce que firent Miller et Nordland [20] et Jerphagnon [21].

Conclusion. - L'analyse détaillée présentée dans cet article est le complément naturel des expériences de mesure des susceptibilités non linéaires menées au laboratoire. L'exploitation des résultats bruts des expériences pose un certain nombre de problèmes et nous avons cherché ici à éclaircir quelques points.

La confrontation entre les expériences et la théorie élaborée a été quotidienne pendant de nombreux mois. Les résultats obtenus après modification des montages suggérée par la théorie (essentiellement la réalisation et l'utilisation d'un laser monomode transverse $\mathrm{TEM}_{00}$ ) sont en très bon accord avec la théorie proposée.

Des résultats expérimentaux sont rassemblés dans un autre article [22], en particulier ceux concernant les propriétés non linéaires des halogénures de cuivre.

Nous tenons à remercier Madame Cautain du Centre de Calcul du C. N. E. T. qui a préparé pour nous les programmes permettant le calcul numérique des flux, et très spécialement Monsieur Duraffourg qui a bien voulu relire et corriger le manuscrit.

\section{ANNEXE 1}

Rappels concernant les modes propres des résonateurs optiques. - Les cavités résonnantes des oscillateurs optiques sont généralement formées de deux miroirs plans ou sphériques dont les axes sont confondus. De telles structures ont été étudiées avec beaucoup de précision, en particulier par Fox et Li [13], Boyd et Gordon [14], Boyd et Kogelnick [15]. Nous nous bornerons à donner un certain nombre de résultats sans démonstration.

Dans une cavité résonnante ouverte, le champ électrique a une direction de propagation qui est l'axe des miroirs, soit oz. Les lignes locales de propagation sont des hyperboles et les surfaces de phase constante des ellipsoïdes orthogonaux à ces hyperboles (voir Fig. 1. $A_{1}$ ). L'amplitude du champ a la forme suivante :

$$
\begin{gathered}
E_{p q}(x, y, z)=E_{o} \frac{W_{o}}{W(z)} H_{p}\left(\frac{x \sqrt{2}}{W(z)}\right) H_{q}\left(\frac{y \sqrt{2}}{W(z)}\right) \\
\times \exp \left(-\frac{x^{2}+y^{2}}{W^{2}(z)}\right) .
\end{gathered}
$$

Dans cette formule, nous avons utilisé les notations définies ci-dessous :

$H_{n}(x)$ : polynôme d'Hermite d'ordre $n$ de la variable $x$, $W(z)$ : diamètre du faisceau à l'ordonnée $z$, égal à

$$
W_{o}\left(1+\frac{4 z^{2}}{b^{2}}\right)^{1 / 2},
$$

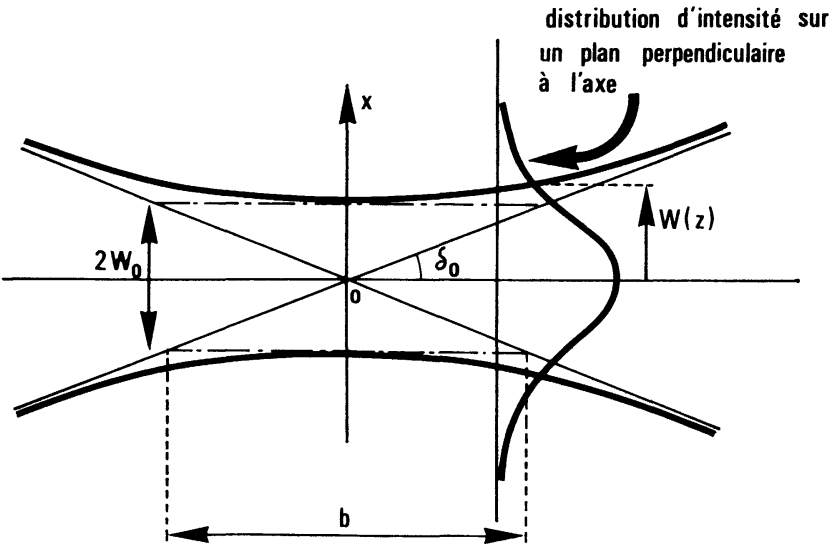

FIG. 1 A-1

$W_{o} \quad:$ diamètre minimum du faisceau $\left(W_{o}^{2}=b \lambda / 2 \pi\right)$, $b$ : paramètre confocal du résonateur défini en fonction de la distance entre miroirs et des rayons de courbure.

Le mode fondamental $p=q=0$ souvent appelé « mode gaussien " est le plus important pour de multiples raisons en optique non linéaire. Nous donnons donc un maximum de résultats le concernant, mais il convient de remarquer que tout ce que nous dirons au sujet des variations de $W(z)$ s'étend sans modification aux modes d'ordres supérieurs (Kogelnick et Li [16]). Pour le «mode gaussien" l'amplitude du champ s'écrit :

$$
E_{o o}(x, y, z)=E_{o} \frac{W_{o}}{W(z)} \exp \left(-\frac{x^{2}+y^{2}}{W^{2}(z)}\right) .
$$

Pour les résonateurs schématisés sur les figures $2 . A_{1}$, on peut montrer que $b^{2}=2 R d-d^{2}$. La transforma-
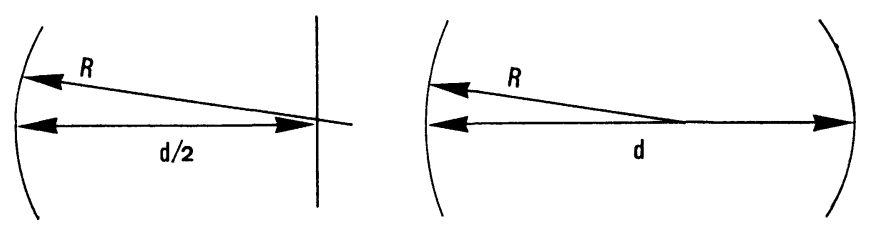

FIG. 2 A-1.

tion d'un faisceau gaussien par une lentille de focale $f$ donne un autre faisceau gaussien dont les paramètres sont :

$$
W_{o}^{\prime}=\frac{f \lambda}{\pi W_{o}} \quad b^{\prime}=\frac{4 f^{2}}{b} .
$$

Lorsqu'un tel faisceau pénètre dans un milieu d'indice $n$, il se transforme en un autre faisceau gaussien de paramètres :

$$
W_{o}^{\prime \prime}=W_{o} \quad ; \quad b^{\prime \prime}=b^{\prime} n
$$

si le matériau est dans la région de focalisation,

$$
W_{o}^{\prime \prime}=\frac{W_{o}^{\prime}}{\sqrt{n}} ; \quad b^{\prime \prime}=b^{\prime}
$$

si le matériau est dans la région de divergence. Comme le montre la figure 2. $A_{1}$, la longueur $b$ repré- 
sente le domaine dans lequel le faisceau gaussien peut être assimilé à un faisceau d'ondes planes. La quasitotalité de l'énergie est située à l'intérieur d'un cylindre de diamètre $2 W_{o}$.
Nous donnons dans le tableau ci-dessous des paramètres typiques des lasers utilisés dans nos expériences et ceux des faisceaux correspondants, justifiant ainsi nos approximations.

\begin{tabular}{|llll}
\hline Laser $\mathrm{CO}_{2}$ & $R=400 \mathrm{~cm} \quad d / 2=250 \mathrm{~cm}$ & $W_{o}=2,55 \mathrm{~mm}$ & $b=388 \mathrm{~cm}$ \\
& Focalisation par une lentille de $20 \mathrm{~cm}$ de focale & $W_{o}^{\prime} \simeq 265 \mu$ & $b^{\prime} \simeq 4 \mathrm{~cm}$ \\
Laser YAG. Nd & $W_{o}=330 \mu$ & $b=65 \mathrm{~cm}$ \\
& $R=100 \mathrm{~cm} \quad d / 2=12 \mathrm{~cm}$ & $W_{o}^{\prime} \simeq 100 \mu$ & $b^{\prime} \simeq 6 \mathrm{~cm}$ \\
& Focalisation par une lentille de $10 \mathrm{~cm}$ de focale & & \\
\hline
\end{tabular}

\section{ANNEXE 2}

Solution particulière des équations de Maxwell avec une source constante. - Ce problème a été étudié par Bloembergen et Pershan [1] ainsi que par Kleinman [17]. Il s'agit de trouver une solution particulière de l'équation ci-dessous :

$$
\bar{\nabla} \times \bar{\nabla} \times \bar{E}+\frac{1}{c^{2}} \stackrel{\varepsilon_{\Omega}}{=} \frac{\partial^{2}}{\partial t^{2}} \bar{E}=-\frac{4 \pi}{c^{2}} \frac{\partial^{2}}{\partial t^{2}} \bar{P}
$$

Dans cette relation $\underline{\underline{\varepsilon_{\Omega}}}=\underline{\underline{I}}+4 \pi \underline{\underline{\chi_{\Omega}}}$ est le ftenseur de permittivité du milieu. La source $\widetilde{\mathrm{P}}$ a la forme suivante :

$$
\bar{P}=\hat{p} P \exp i\left(n \frac{\Omega}{c} \hat{s}-\Omega t\right)
$$

où $n$ est un nombre qui caractérise l'onde de polarisation et n'a $a$ priori aucun rapport avec les indices du milieu à la fréquence $\Omega$. Nous cherchons une solution ayant même phase que $\bar{P}$, donc de la forme :

$$
\bar{E}=\hat{e} E \exp i\left(n \frac{\Omega}{c} \hat{s}-\Omega t\right) .
$$

L'équation (A2.1) s'écrit alors :

$$
\begin{array}{r}
n^{2} I[\hat{s} x(\hat{s} x \hat{e})] E+\varepsilon_{\Omega} \hat{e} E=-4 \hat{p p P} \\
\left(\varepsilon_{\Omega}-n^{2} I\right) \hat{e} E+n^{2} I(\hat{s} \hat{e}) \hat{E s}=-4 \pi \hat{p} P .
\end{array}
$$

Nous définissons le tenseur $\eta$ par la relation : $\eta=\varepsilon_{\Omega}-n^{2} I$ et nous supposerons qu'il est inversible (c'est-à-dire que $n$ n'est pas un des indices du milieu à la fréquence $\Omega$ ). Si l'onde cherchée est transverse $(\hat{s} \perp \hat{e})$, la solution de (A2.3) est évidente; nous sommes ainsi conduits à considérer la partie transverse de $\hat{e}$ et la partie parallèle à $\hat{s}$ et à poser :

$$
\hat{e} E=-4 \pi P \underline{\underline{\eta}}^{-1} \hat{p}+a \underline{\underline{\eta}}^{-1} \hat{s}
$$

où $a$ est un scalaire à déterminer. Si on introduit (A2.4) dans l'équation (A2.3), il vient :

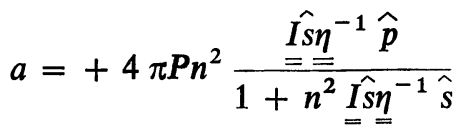

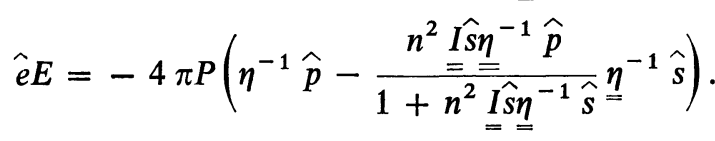

Nous allons développer les calculs dans les deux cas particuliers que nous avons étudiés aux chapitres I et II de la seconde partie.

1) Matériaux isotropes. - Soit $n_{\Omega}$ la valeur de l'indice pour la fréquence considérée $\underline{\underline{\varepsilon_{\Omega}}}=n_{\Omega}^{2} \underline{=}$. La formule (A2.5) s'écrit :

$$
\hat{e} E=-\frac{4 \pi P}{n_{\Omega}^{2}-n^{2}}\left(\hat{p}-\frac{n^{2}}{n_{\Omega}^{2}}(\hat{s} \hat{p}) \hat{s}\right) .
$$

2) MAtÉRiauX UNIAXES. - Soient $n_{o}$ et $n_{e}$ les deux indices du matériau :

$$
\underline{\underline{\varepsilon_{\Omega}}}=\left|\begin{array}{ccc}
n_{o}^{2} & 0 & 0 \\
0 & n_{o}^{2} & 0 \\
0 & 0 & n_{e}^{2}
\end{array}\right| .
$$

La propagation a lieu suivant $\hat{s}=\hat{x} \sin \theta^{\prime}+\hat{z} \cos \theta^{\prime}$. a) Polarisation parallèle à l'axe oy

$$
\begin{gathered}
\hat{p}=\hat{y} \quad n^{2} \hat{I S}_{=}^{-1} \hat{p}=0 \\
\hat{e} E=-\frac{4 \pi P}{n_{o}^{2}-n^{2}} \hat{p} .
\end{gathered}
$$

b) Polarisation parallèle à l'axe ox $\hat{p}=\hat{x}$.

L'indice extraordinaire pour la direction de propagation $\theta^{\prime}$ est tel que :

$$
\frac{1}{n_{e}^{2}\left(\theta^{\prime}\right)}=\frac{\sin ^{2} \theta^{\prime}}{n_{e}^{2}}+\frac{\cos ^{2} \theta^{\prime}}{n_{o}^{2}}
$$

Après un calcul long mais sans difficulté, on peut écrire :

$$
\hat{e} E=-\frac{4 \pi P}{n_{e}^{2}\left(\theta^{\prime}\right)-n^{2}} \frac{n_{e}^{2}\left(\theta^{\prime}\right)}{n_{o}^{2}}\left(\hat{p}-\frac{n^{2}}{n_{e}^{2}}(\hat{s} \hat{p}) \hat{s}\right) \text {. }
$$




\section{ANNEXE 3}

Calcul des flux à la fréquence $2 \omega .-1$ ) RAPPELS DE CERTAINES PROPRIÉTÉS DES FONCTIONS DE BESSEL. -

$$
\begin{array}{ll}
J_{o}(\tau)=\frac{1}{\pi} \int_{o}^{\pi} \exp (i \tau \cos \alpha) \mathrm{d} \alpha & t J_{1}(t)=\int_{o}^{t} \tau J_{o}(\tau) \mathrm{d} \tau \\
J_{o}(\tau)=J_{o}(-\tau) & J_{1}\left(t^{*}\right)=J_{1}^{*}(t) .
\end{array}
$$

Nous définissons des fonctions auxiliaires par les formules suivantes :

$$
\begin{gathered}
F(t)=2-\frac{J_{l}(t)}{t} \\
F^{+}(t)=\frac{1}{2}\left[F(t)+F\left(t^{*}\right)\right]=R_{e}(F(t)) \\
F^{-}(t)=\frac{1}{2 i}\left(F(t)-F\left(t^{*}\right)\right)=I_{m}(F(t)) .
\end{gathered}
$$

Les variables $\tau$ et $t$ sont réelles ou complexes. La courbe représentative de la fonction $F(t)$ pour des valeurs réelles de la variable comprises entre 0 et 10 , est donnée à la figure $1 . \mathrm{A}_{3}$.

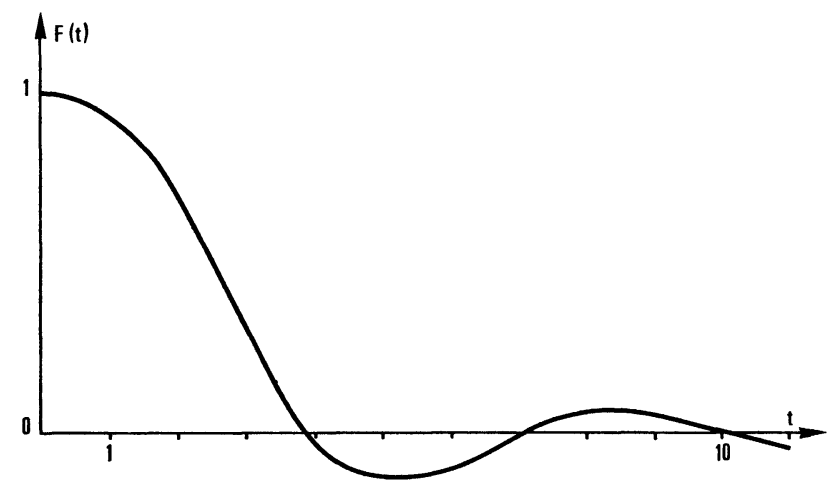

Fig. 1 A-4.

2) Calcul des intégrales INTERvenant dans les FLUX. - 1. Milieux transparents. Dans l'approximation que nous avons faite, toutes les variables indicées $\omega$ et celles indicées $2 \omega$ sont confondues dans toutes les expressions où elles n'apparaissent pas sous forme de différences. La surface $\Sigma_{I}$ à travers laquelle le faisceau sort du prisme est une ellipse de centre $\Omega$ et d'axe $2 W_{o}$ et $\left(2 W_{o} / \cos \theta\right) \cdot\left(\cos \theta^{\prime} / \cos \varphi^{\prime}\right)$. Considérons la direction $\Omega z^{\prime}$ faisant un angle $\psi$ avec la normale au deuxième dioptre, tel que

$$
\cos \psi=\cos \theta \frac{\cos \varphi^{\prime}}{\cos \theta^{\prime}} .
$$

Le cylindre de génératrice parallèle à $\Omega z^{\prime}$ s'appuyant sur $\Sigma_{\mathrm{l}}$, a pour section droite un cercle de diamètre $2 W_{o}$. (Au minimum de déviation ce cylindre est confondu avec le pinceau lumineux sortant du prisme.) Nous effectuons nos calculs dans le système de coordonnées cylindriques d'axe $\Omega z^{\prime}$, et nous utiliserons comme intermédiaire le système $\Omega x^{\prime} z^{\prime} y^{\prime}$ (oy//oy', $\widehat{\mathrm{o} z, \mathrm{oz}^{\prime}}=\psi-S$ ), voir figure $2 . \mathrm{A}_{3}$. Dans ce système, l'équation du deuxième dioptre est $z^{\prime}=-x^{\prime} \operatorname{tg} \psi$, l'élément de surface sur ce plan est $\mathrm{d} \sigma=\rho(\mathrm{d} \rho \mathrm{d} \alpha / \cos \psi)$,

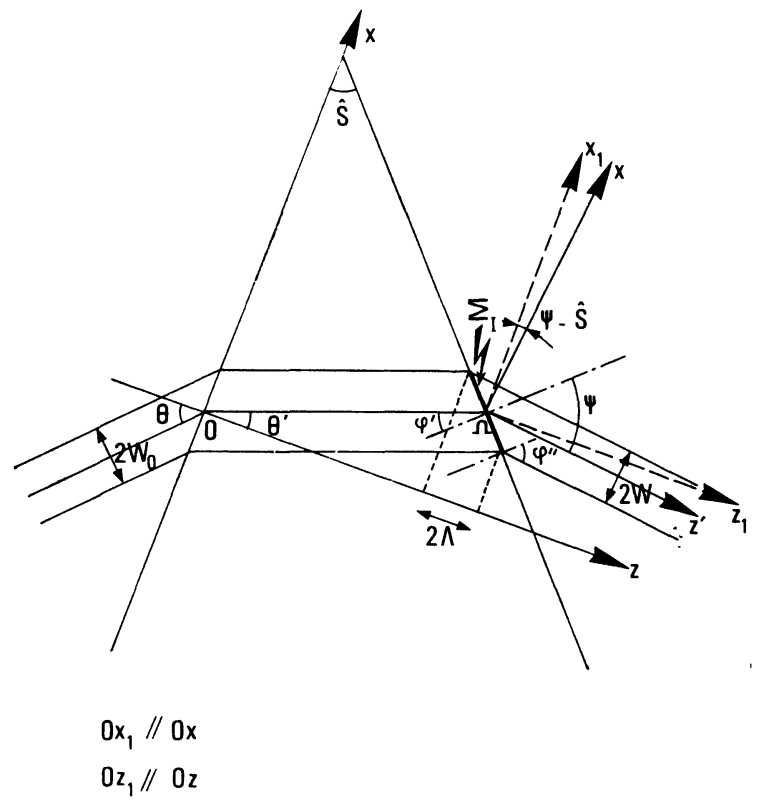

FIG. 2 A-4.

et $z=L-(\sin s / \cos \psi) \rho \cos \alpha$, où L est la cote de $\Omega$. D'après (30), le vecteur de Poynting $\bar{S}_{T}$ se présente sous la forme d'une somme de trois termes; on peut donc décomposer le flux $\Phi_{2 \omega}$ en trois parties :

$$
\begin{aligned}
& \Phi_{l}=\frac{c}{8 \pi}\left(D t_{\omega}^{2} T_{2 \omega} A_{l} E_{\omega}^{2}\right)^{2} \cos \varphi^{\prime \prime} \int_{\Sigma_{I}} \rho \frac{\mathrm{d} \rho \mathrm{d} \alpha}{\cos \psi} \\
& \Phi_{L}=\frac{c}{8 \pi}\left(D t_{\omega}^{2} T_{\omega} E_{\omega}^{2}\right)^{2} \cos \varphi^{\prime \prime} \int_{\Sigma_{I}} \rho \frac{\mathrm{d} \rho \mathrm{d} \alpha}{\cos \psi} \\
& \Phi_{I}=\frac{c}{8 \pi}\left(D t_{\omega}^{2} E_{\omega}^{2}\right)^{2} 2 \cos \varphi^{\prime \prime} A_{l} T_{\omega} T_{2 \omega} \\
& \times \int_{\Sigma_{I}} \cos \left(\frac{\pi}{l_{c}} z\right) \rho \frac{\mathrm{d} \rho \mathrm{d} \alpha}{\cos \psi} .
\end{aligned}
$$

Les intégrales intervenant dans $\Phi_{L}$ et $\Phi_{l}$ sont égales à $\pi W^{2} / \cos \psi$. Le calcul du flux d'interférence se ramène à celui de l'intégrale suivante :

$$
I=\int_{o}^{W o} \int_{o}^{2 \pi} \cos \frac{\pi}{l_{c}}\left(L-\frac{\sin S}{\cos \psi} \rho \cos \alpha\right) \rho \mathrm{d} \rho \mathrm{d} \alpha .
$$

Nous utilisons les relations suivantes :

$$
\begin{gathered}
\int_{o}^{2 \pi} \sin \left(\frac{\pi}{l_{c}} \frac{\sin S}{\cos \psi} \rho \cos \alpha\right) \mathrm{d} \alpha=0 \\
\int_{o}^{2 \pi} \cos \left(\frac{\pi}{l_{c}} \frac{\sin S}{\cos \psi} \rho \cos \alpha\right) \mathrm{d} \alpha=2 \pi J_{o}\left(\frac{\pi}{l_{c}} \frac{\sin S}{\cos \psi} \rho\right) \\
2 \pi \int_{o}^{W o} J_{o}\left(\frac{\pi \sin S}{l_{c} \cos \psi} \rho\right) \rho \mathrm{d} \rho=2 \pi W_{o}^{2} \frac{J_{1}\left(\frac{\pi \Lambda}{l_{c}}\right)}{\frac{\pi \Lambda}{l_{c}}} .
\end{gathered}
$$


Le paramètre $\Lambda=W_{o}(\sin S / \cos \psi)$ a une interprétation physique immédiate ; $2 \Lambda$ est la projection du grand axe de $\Sigma_{I}$ sur oz, c'est donc la variation de cote sur l'extension du faisceau. Les trois termes du flux s'écrivent :

$$
\begin{aligned}
\Phi_{l}= & \frac{c}{8 \pi}\left(D t_{\omega}^{2} E_{\omega}^{2} A_{l} T_{2 \omega}\right)^{2} \frac{\cos \varphi^{\prime \prime}}{\cos \psi} \pi W_{o}^{2} \\
\Phi_{L}= & \frac{c}{8 \pi}\left(D t_{\omega}^{2} E_{\omega} T_{\omega}\right)^{2} \frac{\cos \varphi^{\prime \prime}}{\cos \psi} \pi W_{o}^{2} \\
\Phi_{I}= & \frac{c}{8 \pi}\left(D t_{\omega}^{2} E_{\omega}^{2}\right)^{2} A_{l} T_{2 \omega} T_{\omega} \frac{2 \cos \varphi^{\prime \prime}}{\cos \psi} \\
& \times \pi W_{o}^{2} F\left(\frac{\pi \Lambda}{l_{c}}\right) \cos \left(\frac{\pi L}{l_{c}}\right) .
\end{aligned}
$$

Au minimum de déviation $\psi=\varphi^{\prime \prime}=\theta$. Nous introduisons les infiniment petits $\varepsilon, \eta$ et $\beta$ par les relations :

$$
\begin{aligned}
& T=T_{2 \omega}=T_{\omega}(1+\varepsilon) \quad A_{l}+1=\eta \\
& \cos \varphi^{\prime \prime}=\cos \varphi_{2 \omega}^{\prime \prime}=(1+2 \beta) \cos \varphi_{\omega}^{\prime \prime}
\end{aligned}
$$

$$
\begin{gathered}
\Phi_{2 \omega}=\frac{c}{8 \pi}\left(D t_{\omega}^{2} T E_{\omega}^{2}\right)^{2} 2 \pi W_{o}^{2}(1+\eta+\beta+\varepsilon) \\
\times\left(1-F\left(\frac{\pi \Lambda}{l_{c}}\right) \cos \left(\frac{\pi L}{l_{c}}\right)\right)
\end{gathered}
$$

2. Milieux absorbants. Dans l'annexe $\mathrm{n}^{\circ} 4$, nous montrons que pour des matériaux légèrement transparents dont l'indice complexe est $\stackrel{\circ}{n}=n(1+i \kappa)$, la phase d'une onde plane s'écrit :

$$
i \bar{k}^{\prime} \bar{r}=i \frac{\Omega}{c}\left(n x \sin \theta^{\prime}+n z \cos \theta^{\prime}\right)-\frac{\Omega}{c} \frac{n \kappa}{\cos \theta^{\prime}} z .
$$

Le vecteur de Poynting en un point du deuxième dioptre s'exprime par la formule (42) :

$$
\begin{aligned}
\bar{S}(R)= & \frac{c}{8 \pi}\left(D t_{\omega}^{2} E_{\omega}^{2}\right)^{2}\left[s_{L}^{\prime \prime} T_{\omega}^{2} \exp \left(-2 \gamma_{\omega} z\right)+\right. \\
& +\hat{s}_{l}^{\prime \prime} T_{2 \omega}^{2} A_{l}^{2} \exp \left(-\gamma_{2 \omega} z\right)+\left(\hat{s}_{L}^{\prime \prime}+\hat{s}_{l}^{\prime \prime}\right) T_{\omega} T_{2 \omega} A_{l} \\
& \left.\times \exp \left(-\left(\gamma_{\omega}+\frac{1}{2} \gamma_{2 \omega}\right) z\right) \cos \left(\frac{\pi z}{l_{c}}\right)\right]
\end{aligned}
$$

Les coefficients d'absorption efficace $\gamma_{\omega}$ et $\gamma_{2 \omega}$ sont définis en annexe $n^{\circ} 4$. Sur la surface $\Sigma_{I}$ :

$$
z=L-\frac{\sin S}{\cos \psi} \rho \cos \alpha .
$$

On peut comme au paragraphe précédent décomposer le flux $\Phi_{2 \omega}$ en trois parties

$$
\begin{aligned}
\Phi_{l}= & \frac{c}{8 \pi}\left(D t_{\omega}^{2} T_{2 \omega}^{2} A_{l} E_{\omega}^{2}\right)^{2} \cos \varphi^{\prime \prime} \exp \left(-\gamma_{2 \omega} L\right) \int_{\Sigma_{I}} \exp \left(\gamma_{2 \omega} \frac{\sin S}{\cos \psi} \rho \cos \alpha\right) \frac{\rho \mathrm{d} \rho \mathrm{d} \alpha}{\cos \psi} \\
\Phi_{L}= & \frac{c}{8 \pi}\left(D t_{\omega}^{2} T_{\omega} E_{\omega}^{2}\right)^{2} \cos \varphi^{\prime \prime} \exp \left(-2 \gamma_{\omega} L\right) \int_{\Sigma_{I}} \exp \left(2 \gamma_{\omega} \frac{\sin S}{\cos \psi} \rho \cos \alpha\right) \frac{\rho \mathrm{d} \rho \mathrm{d} \alpha}{\cos \psi} \\
\Phi_{I}= & \frac{c}{8 \pi}\left(D t_{\omega}^{2} E_{\omega}^{2}\right)^{2} 2 \cos \varphi^{\prime \prime} A_{l} T_{\omega} T_{2 \omega} \exp \left(-\left(\gamma_{\omega}+\frac{1}{2} \gamma_{2 \omega}\right) L\right) \\
& \times \int_{\Sigma_{I}} \exp \left(\left(\gamma_{\omega}+\frac{1}{2} \gamma_{2 \omega}\right) \frac{\sin S}{\cos \psi} \rho \cos \alpha\right) \cos \left(\frac{\pi}{l_{c}}\left(L-\rho \cos \alpha \frac{\sin S}{\cos \psi}\right)\right) \frac{\rho \mathrm{d} \rho \mathrm{d} \alpha}{\cos \psi}
\end{aligned}
$$

Les intégrales sont du même type que celles calculées au paragraphe précédent mais avec des arguments complexes. On trouve que :

$$
\begin{aligned}
\Phi_{l}= & \frac{c}{8 \pi}\left(D T_{2 \omega} A_{l} t_{\omega}^{2} E_{\omega}^{2}\right)^{2} \frac{\cos \varphi^{\prime \prime}}{\cos \psi} \pi W_{o}^{2} \exp \left(-\gamma_{2 \omega} L\right) F\left(-i \gamma_{2 \omega} \Lambda\right) \\
\Phi_{L}= & \frac{c}{8 \pi}\left(D T_{\omega} t_{\omega}^{2} E_{\omega}^{2}\right)^{2} \frac{\cos \varphi^{\prime \prime}}{\cos \psi} \pi W_{o}^{2} \exp \left(-2 \gamma_{\omega} L\right) F\left(-2 i \gamma_{\omega} \Lambda\right) \\
\Phi_{I}= & \frac{c}{8 \pi}\left(D t_{\omega}^{2} E_{\omega}^{2}\right)^{2} A_{l} T_{\omega} T_{2 \omega} \frac{2 \cos \varphi^{\prime \prime}}{\cos \psi} \pi W_{o}^{2} \exp \left(-\left(\gamma_{\omega}+\frac{1}{2} \gamma_{2 \omega}\right) L\right) \\
& \times\left[\cos \frac{\pi L}{l_{c}} F^{+}\left(\left(\frac{\pi}{l_{c}}-i\left(\gamma_{\omega}+\frac{1}{2} \gamma_{2 \omega}\right)\right) \Lambda\right)+\sin \frac{\pi L}{l_{c}} F^{-}\left(\left(\frac{\pi}{l_{c}}-i\left(\gamma_{\omega}+\frac{1}{2} \gamma_{2 \omega}\right)\right) \Lambda\right)\right] .
\end{aligned}
$$

Lorsqu'on se place au minimum de déviation et que l'on introduit les mêmes notations qu'au paragraphe précédent, il vient : 


$$
\begin{aligned}
\Phi_{2 \omega}=\frac{c}{8 \pi}\left(D t_{\omega}^{2} T E_{\omega}^{2}\right)^{2} \pi W_{o}^{2}(1+\eta+\beta+\varepsilon)\left\{\exp \left(-\gamma_{2 \omega} L\right) F\left(-i \gamma_{2 \omega} \Lambda\right)+\exp \left(-2 \gamma_{\omega} L\right) F\left(-2 i \gamma_{\omega}\right)\right. \\
-2 \exp \left(-\left(\gamma_{\omega}+\frac{1}{2} \gamma_{2 \omega}\right) L\right)\left(\cos \frac{\pi L}{l_{c}} F^{+}\left(\left(\frac{\pi}{l_{c}}-i\left(\gamma_{\omega}+\frac{1}{2} \gamma_{2 \omega}\right)\right) \Lambda\right)\right. \\
\left.\left.+\sin \frac{\pi L}{l_{c}} F^{-}\left(\left(\frac{\pi}{l_{c}}-i\left(\gamma_{\omega}+\frac{1}{2} \gamma_{2 \omega}\right)\right) \Lambda\right)\right)\right\}
\end{aligned}
$$

Dans la plupart des expériences, on a utilisé des faisceaux focalisés de sorte que $\Lambda$ (inférieur à $100 \mu$ ) est assez petit pour que $\gamma \Lambda$ soit négligeable devant l'unité.

De plus, pour les longueurs de cohérence généralement rencontrées $\pi / l_{c} \gg \gamma$, si bien que la formule cidessus peut en pratique être remplacée par :

$$
\begin{aligned}
\Phi_{2 \omega}=\frac{c}{8 \pi} & \left(D t_{\omega}^{2} T E_{\omega}^{2}\right)^{2} \pi W_{o}^{2} \\
& \times\left(\exp \left(-\gamma_{2 \omega} L\right)+\exp \left(-2 \gamma_{\omega} L\right)\right. \\
& -2 \exp \left(-\left(\gamma_{\omega}+\frac{1}{2} \gamma_{2 \omega}\right) L\right) \\
& \left.\times \cos \frac{\pi L}{l_{c}(\theta)} F\left(\frac{\pi \Lambda}{l_{c}(\theta)}\right)\right)
\end{aligned}
$$

qui est d'un emploi beaucoup plus commode.

\section{ANNEXE 4}

Rappels concernant l'optique des matériaux absorbants. - On trouve dans de nombreux traités d'optique (par exemple Born et Wolf [19]) la théorie complète de l'électromagnétisme dans les milieux absorbants. Nous nous contenterons de rappeler quelques résultats nécessaires au développement du chapitre III de la deuxième partie.

Les matériaux absorbants sont caractérisés par une atténuation exponentielle de l'intensité lumineuse pénétrant le milieu. On peut décrire l'optique de ces corps tout en conservant formellement les lois démontrées pour les matériaux transparents (Landau, Lifchitz [18]) en généralisant la notion d'indice qui est alors une grandeur complexe ; nous écrivons ainsi :

$$
\stackrel{\circ}{n}=n(1+i \kappa) \text {. }
$$

RÉFRACTION D'UNE ONDE PLANE PAR UN DIOPTRE VIDE - MATÉRIAU ABSORBANT. - Une onde plane passe $\mathrm{du}$ vide où elle est représentée par $\bar{E}(r, t)=\widehat{e} E \exp i(\overrightarrow{k r}-\Omega t)$, dans le milieu absorbant où $\bar{E}^{\prime}(r, t)=\widehat{e}^{\prime} E^{\prime} \exp i\left(\overline{k^{\prime}} \bar{r}-\Omega t\right)$, avec :

$$
\bar{k}^{\prime}=\frac{\Omega}{c} \hat{s} \quad \text { et } \quad \bar{k}^{\prime}=\stackrel{\circ}{n} \frac{\Omega}{c} \hat{s}^{\prime} .
$$

Bien que les vecteurs $\bar{k}^{\prime}$ et $\widehat{s}^{\prime}$ soient des grandeurs complexes, la traversée du dioptre s'effectue toujours de manière à ce que $\bar{k}_{T}=\bar{k}_{T}^{\prime}$. De plus, on peut toujours choisir les axes tels que $k_{y}=k_{y}^{\prime}=0$, de sorte que :

$s_{x}=\sin \theta \quad s_{x}^{\prime}=\sin \theta^{\prime}=\frac{1}{n} \sin \theta$

$s_{y}=0 \quad s_{y}^{\prime}=0$

$s_{\mathrm{z}}=\cos \theta \quad s_{\mathrm{z}}^{\prime}=\cos \theta^{\prime}=\frac{1}{\stackrel{\circ}{n}} \cos \theta\left(1+\frac{\stackrel{\circ}{n}^{2}-1}{\cos ^{2} \theta}\right)^{1 / 2}$.

La variation spatiale de la phase de l'onde réfractée peut se mettre sous la forme suivante :

$\bar{k}^{\prime} \bar{r}=\frac{\Omega}{c}\left[x \sin \theta+z \cos \theta\left[1+\frac{\stackrel{\circ}{ }^{2}-1}{\cos ^{2} \theta}\right]^{1 / 2}\right]$

où l'on peut remarquer que l'influence de la partie complexe de l'indice se fait sentir sur la variation suivant oz seulement. Si $\stackrel{n}{n}$ est réel, on retrouve les lois habituelles. Il est commode de définir l'angle $\tau^{\prime}$ par la relation :

$$
\left[1+\frac{\stackrel{\circ}{ }^{2}-1}{\cos ^{2} \theta}\right]^{1 / 2}=\frac{n}{\cos \theta}\left[\cos \tau^{\prime}+\frac{i \kappa}{\cos \tau^{\prime}}\right] .
$$

L'angle $\tau^{\prime}$ est toujours réel ; en effet, il est défini par son cosinus, lui-même déterminé par l'équation bicarrée (A4.3) dont les solutions sont toujours réelles et comprises entre 0 et 1 .

$\cos ^{4} \tau^{\prime}-\left[1-\kappa^{2}-\frac{\sin ^{2} \theta}{n^{2}}\right] \cos ^{2} \tau^{\prime}-\kappa^{2}=0$.

Alors :

$\bar{k}^{\prime} \bar{r}=\frac{\Omega}{c}\left[x \sin \theta+z n \cos \tau^{\prime}+i z \frac{n \kappa}{\cos \tau^{\prime}}\right]$.

Les surfaces équi-amplitude sont des plans parallèles au plan du dioptre. Les surfaces équi-phase sont des plans dont la direction des normales fait avec oz un angle dont la tangente est $\sin \theta / n \cos \tau^{\prime}$.

Les formules de Fresnel se dérivent de la même manière que pour les matériaux transparents. Mais les facteurs de réflexion $R$ et de transmission $T$ sont des grandeurs complexes. Dans le cas d'une polarisation perpendiculaire au plan d'incidence, on peut écrire : 


$$
\begin{aligned}
R=r e^{i \eta} & =\frac{\cos \theta-\left[n \cos \tau^{\prime}+i \frac{n \kappa}{\cos \tau^{\prime}}\right]}{\cos \theta+\left[n \cos \tau^{\prime}+i \frac{n \kappa}{\cos \tau^{\prime}}\right]} \\
T=t e^{i \chi} & =\frac{2 \cos \theta}{\cos \theta+\left[n \cos \tau^{\prime}+i \frac{n \kappa}{\cos \tau^{\prime}}\right]} \\
\mathrm{t}^{2} & =\frac{4 \cos { }^{2} \theta}{\frac{n^{2} \kappa^{2}}{\cos ^{2} \tau^{\prime}}+\left(\cos \theta+n \cos \tau^{\prime}\right)^{2}} \\
\operatorname{tg} \chi & =\frac{n \kappa}{\cos \tau^{\prime}\left(\cos \theta+n \cos \tau^{\prime}\right)} .
\end{aligned}
$$

Cas des matériaux légèrement absorbants.

$\kappa$ est petit devant l'unité et ses puissances sont négligeables.

$$
\cos ^{2} \tau^{\prime} \simeq 1-\frac{\sin ^{2} \theta}{n^{2}}+\mathrm{O}\left(\kappa^{2}\right)
$$

Si l'absorption était nulle, l'angle de réfraction $\theta^{\prime}$ serait tel que : $\sin \theta^{\prime}=\sin \theta / n$. Donc $\tau^{\prime} \simeq \theta^{\prime}$ et : $\exp \left(i \bar{k}^{\prime} \bar{r}\right)=$

$=\exp \left[i \frac{\Omega}{c}\left(n x \sin \theta^{\prime}+n z \cos \theta^{\prime}\right)-\frac{\Omega}{c} \frac{n \kappa}{\cos \theta^{\prime}} z\right]$.

Si l'on considère la propagation de la phase, tout se passe comme si le matériau était transparent. Par contre l'amplitude de l'onde est une exponentielle décroissante en fonction de la profondeur de pénétration.

Le coefficient d'absorption efficace est :

$$
\gamma_{\Omega}=\frac{2 \Omega}{c} \frac{n \kappa}{\cos \theta^{\prime}} .
$$

Le facteur de transmission est :

$$
t=\frac{2 \cos \theta^{\prime}}{\cos \theta+n \cos \theta^{\prime}}
$$

Pour de tels matériaux, les lois de l'optique des milieux transparents sont conservées, la seule modification appréciable est due à l'atténuation exponentielle de l'amplitude de l'onde.

\section{ANNEXE 5}

Conditions aux limites pour des ondes extraordinaires. - Dans cette annexe, nous étendons le calcul du chapitre I concernant les conditions aux limites à la surface d'un dioptre au cas des ondes extraordinaires. On suppose le dioptre normal à l'axe optique du matériau.
Le rayonnement fondamental induit dans le diélectrique une ou plusieurs ondes forcées à la fréquence $2 \omega$. Comme dans les cas précédents l'écriture des conditions aux limites fixe l'amplitude d'une onde réfléchie et d'une onde libre dans le milieu. Soit $\rho_{j}$ l'angle de la direction de propagation de la phase et de la direction de propagation de l'énergie pour la «j-ième » onde. S'il s'agit d'une onde forcée, $\rho_{j}$ est donné par le tenseur de susceptibilité du deuxième ordre du matériau et les indices, s'il s'agit d'une onde libre, par les indices seulement ; dans ce cas, en appelant $\alpha$ l'angle de l'axe optique et de la direction de propagation de la phase :

$$
\operatorname{tg} \rho_{j}=\frac{n_{j}^{e 2}-n_{j}^{o 2}}{n_{j}^{o 2} \operatorname{tg} \alpha+n_{j}^{e 2} \operatorname{cotg} \alpha} .
$$

Les composantes des vecteurs polarisation et des vecteurs unitaires suivant la direction de propagation des phases sont données dans le tableau ci-dessous.

$$
\begin{array}{ccccc} 
& \hat{e}_{R} & \hat{s}_{R} & \hat{e}_{j}^{\prime} & \frac{\hat{s}_{j}^{\prime}}{\sin \theta} \\
x & -\cos \theta & \cos \left(\theta_{j}^{\prime}+\rho_{j}\right) & \sin \theta_{j}^{\prime} \\
y & 0 & 0 & 0 & 0 \\
z & -\sin \theta & -\cos \theta & -\sin \left(\theta_{j}^{\prime}+\rho_{j}\right) & \cos \theta_{j}^{\prime}
\end{array}
$$

La continuité des composantes tangentielles des champs s'écrit :

$$
\begin{aligned}
E_{R} e_{R, x} & =\sum_{j} e_{j, x}^{\prime} E_{j}^{\prime} \\
E_{R} e_{R, y} & =\sum_{j} e_{j, y}^{\prime} E_{j}^{\prime} \\
\left(s_{R, y} e_{R, z}-s_{R, z} e_{R, y}\right) E_{R} & =\sum_{j}\left(s_{j, y}^{\prime} e_{j, z}^{\prime}-s_{j, z}^{\prime} e_{j, y}^{\prime}\right) n_{j} E_{j}^{\prime} \\
\left(s_{R, z} e_{R, x}-s_{R, x} e_{R, z}\right) E_{R} & =\sum_{j}\left(s_{j, z}^{\prime} e_{j, x}^{\prime}-s_{j, x}^{\prime} e_{j, z}^{\prime}\right) n_{j} E_{j} .
\end{aligned}
$$

Avec des polarisations et des directions de propagation choisies, les relations (A5.2) et (A5.3) sont automatiquement vérifiées; les deux autres relations s'écrivent :

$$
\begin{aligned}
-E_{R} \cos \theta & =\sum_{j} E_{j}^{\prime} \cos \left(\theta_{j}^{\prime}+\rho_{j}\right) \\
E_{R} & =+\sum_{j} n_{j} E_{j}^{\prime} \cos \rho_{j} .
\end{aligned}
$$

On cherche l'amplitude de l'onde libre $E_{l}$ qui est dans le matériau la seule inconnue puisque les autres ondes sont définies par les sources.

$$
E_{l}=-\sum_{j \neq l} \frac{\cos \left(\theta_{j}^{\prime}+\rho_{j}\right)+n_{j} \cos \theta \cos \rho_{j}}{\cos \left(\theta_{l}^{\prime}+\rho_{l}\right)+n_{l} \cos \theta \cos \rho_{l}} E_{j}^{\prime}
$$




\section{Bibliographie}

[1] Bloembergen (N.), Pershan (P. S.), Phys. Rev., 1962, $128,606$.

[2] Boyd (G. D.), Ashrin (A.), Dziedzic (J. M.), KLeINMAN (D. A.), Phys. Rev. 1965, 137A, 1305.

[3] Kleinman (D. A.), Ashkin (A.), Boyd (G. D.). Phys. Rev., 1966, 145, 338.

[4] Boyd (G. D.), Kleinman (D. A.), J. Appl. Phys. 1968, 39, 3597.

[5] Bjorkholm (J. E.), Phys. Rev., 1966, 142, 128.

[6] Jerphagnon (J.), Kurtz (S. K.), J. Appl. Phys., 1970, 41, 1667.

[7] Maker (P. D.), Terhune (R. W.), Nisenoff (N.), Savage (C. M.), Phys. Rev. Letters, 1962, 8, 21.

[8] Jerphagnon (J.), Thèse, Université de Paris (1967).

[9] Bloembergen (N.), Simon (H. J.), Lee (C. H.), Phys. Rev., 1969, 181, 1261.

[10] Miller (R. C.), Kleinman (D. A.), Savage (C. M.), Phys. Rev. Letters, 1963, 11, 1464.

[11] Afans'ev (A. M.), Manykin (E. A.), Sov. Phys. JETP, 1965, 21, 323.
[12] Soref (R. A.), Moos (H. W.), J. Appl. Phys., 1965, $35,2152$.

[13] Fox (A. G.), Li (T.), B. S. T. J., 1961, 40, 453.

[14] Gordon (G. P.), Boyd (G. D.), B. S. T. J., 1961. 40, 489.

[15] Boyd (G. D.), Kogelnik (H.), B. S. T.J., 1962, 41, 1347.

[16] Kogelnik (H.), Li (T.), Appl. Optics, 1966, 5, 1550.

[17] Kleinman (D. A.), Phys. Rev., 1962, 128, 1761.

[18] LandaU, Lifchitz, Electromagnétisme des Milieux Continus (Editions de la Paix).

[19] Born (M.), Wolf (E.), Principles of Optics (Pergamon Press).

[20] Miller (R. C.), Nordland (W. A.), Appl. Phys. Letters, 1970, 16, 174.

[21] Jerphagnon (J.), Appl. Phys. Letters, 1970, 16, 298.

[22] Chemla (D.), KupeceK (P.), Schwartz (C.), Schwab (C.), GoldTzene (A.), à paraître, dans I. E. E. E. J. of Quantum Electronics, mars 1971. 